\title{
A Parallel, 2-DOF Exoskeleton for the Human Shoulder: Device Characterization and Preliminary Results on Healthy Subjects
}

Rainier Natividad ( $\nabla$ rfnatividad@u.nus.edu)

National University of Singapore Faculty of Engineering https://orcid.org/0000-0002-7143-1874

Tiana Miller-Jackson

National University of Singapore

Chen-Hua, Raye Yeow

National University of Singapore

\section{Research}

Keywords: Fabric Actuators, Shoulder Exosuit, Shoulder Exoskeleton, Soft Exoskeleton, Pneumatic Bending Actuator, Soft Robotic Actuator, Wearable Robot, Muscle Activation

Posted Date: May 26th, 2020

DOI: https://doi.org/10.21203/rs.3.rs-30545/v1

License: (c) (i) This work is licensed under a Creative Commons Attribution 4.0 International License. Read Full License 


\section{A Parallel, 2-DOF Exoskeleton for the Human Shoulder: Device Characterization and Preliminary Results on Healthy Subjects}

1 Rainier F. Natividad1,2, Tiana Miller-Jackson1,2, Raye Yeow Chen-Hua1,2,3*

2 1Evolution Innovation Lab, Department of Biomedical Engineering, National University of

3 Singapore (NUS), Singapore

4 2Advanced Robotics Centre (ARC), National University of Singapore (NUS), Singapore

5 3Singapore Institute of Neurotechnology (SINAPSE), National University of Singapore (NUS),

6 Singapore

7

8 * Correspondence:

9 Raye Yeow Chen-Hua

10 bieych@nus.edu.sg

11 Keywords: Fabric Actuators, Shoulder Exosuit, Shoulder Exoskeleton, Soft Exoskeleton,

12 Pneumatic Bending Actuator, Soft Robotic Actuator, Wearable Robot, Muscle Activation.

141 Abstract

\section{$15 \quad 1.1 \quad$ Background}

16 Humans are highly reliant on the efficient function of their upper limb. Neuromuscular disorders that

17 impair the function of the shoulder consequently reduce quality of life. Robotic rehabilitation serves

18 as an attractive treatment choice due to its promising results and its ability to alleviate the demands on 
19 therapists and clinicians. Nevertheless, current robotic architectures are not optimized for the human

20 shoulder but are more apt for industrial environments. Pneumatically powered soft robotic actuators

21 present an attractive method to create shoulder exoskeletons due to their compliance and relatively low

22 mass. However, current actuators lack the necessary functions to provide support to the entire

23 shoulder's range of motion.

\section{$24 \quad 1.2$ Methods}

25 A modular, fabric pneumatic actuator was constructed. The actuator design allows it to perform three-

26 dimensional (3-D) bends with minimal resistance. Four actuators were combined to create a soft

27 shoulder exoskeleton. Each actuator drives one direction of motion: elevation and depression, rotation

28 of the plane of elevation. The torque output of the actuator was measured using a customized two-axis

29 torque measurement system. Exoskeleton functionality was tested through surface electromyography

30 of relevant shoulder muscles. 10 healthy subjects were recruited and performed arm motions under the

31 assistance of the exoskeleton.

\section{$\begin{array}{lll}32 & 1.3 & \text { Results }\end{array}$}

33 The actuator can reach full bending $\left(>360^{\circ}\right)$ with low pressures $(\sim 10 \mathrm{kPA})$. Its torque output is highly

34 dependent on its geometry. Moreover, torque output is reduced as the bending angles increase. The

35 actuators installed on the exoskeleton output $11.15 \mathrm{~N}-\mathrm{m}$ of torque at the neutral position, and $4.44 \mathrm{~N}-\mathrm{m}$

36 at $90^{\circ}$ shoulder elevation. The test on healthy subjects showed that use of the exoskeleton reduces

37 muscle activation by up to $65 \%$ when performing shoulder elevation, and up to $34 \%$ when rotating the

38 plane of elevation. Use of the exoskeleton also resulted in a change in arm trajectory when performing

39 elevation and depression movements.

\section{$40 \quad 1.4$ Conclusions}


41 The reduction in muscle activation highlights the ability of a soft-robotic exoskeleton in supporting

42 arm movements. Moreover, the presented exoskeleton design successfully demonstrated its ability to

43 generate two degree-of-freedom support for the human shoulder

\section{Background}

45 The human arm is responsible for a majority of movements necessary for activities of daily living

46 (ADL) (1). As a result, humans are extremely reliant on their arms in order to live an optimal life. The

47 shoulder acts as the base of the human arm and grants the arm with maximum mobility due to the

48 shoulders nearly spherical range of motion. It is imperative to restore proper mobility to the shoulder

49 when its function is impaired (2). Clinical upper-limb exoskeletons are used as an adjunct to treat

50 disorders that inhibit shoulder movement $(3,4)$. Typically, these exoskeletons are constructed using

51 techniques pioneered in industrial robotics. Rigid electric motors are placed concentrically with the

52 humeral head while rigid links are attached parallel to the humerus, connecting the motors to the arm.

53 The shoulder joint, also known as the shoulder complex, is typically oversimplified as a ball-and-socket

54 joint $(5,6)$. In reality, the shoulder complex is a system of multiple joints: the scapulothoracic joint,

55 the sternoclavicular joint, the acromioclavicular joint, and the glenohumeral $(\mathrm{GH})$ joint. These joints

56 connect the sternum, clavicle, scapula and humerus to form the shoulder complex. The four joints move

57 in concert, constantly translating the location of the humeral head during shoulder motion (7). This

58 motion is called scapulohumeral rhythm. Robotic shoulder exoskeletons must therefore account for the

59 unique movement of the shoulder complex (8), typically by translating the position of the motors during

60 shoulder movement (5). Alternatively, designers also employ an additional link in order to account for

61 translation of the humeral head. However, the addition of such a link increases the likelihood of joint

62 misalignment (9). Moreover, these designs weigh substantially heavier relative to the weight of a

63 human, which adds unwanted inertia to the arm and restricts the mobility of the user. 
64 In contrast to traditional robotic designs, soft robotics utilizes naturally flexible materials, both as prime

65 movers and structural links. This archetype of robotics behaves similar to continuum structures (10) as

66 compared to a system of rigid bodies that typically characterize traditional robots. The distinct

67 compliance and usage of non-rigid power transmission elements have enabled soft robots to more

68 closely emulate the movement of animals. They are uniquely suited to power robotic exoskeletons due

69 to their compliance, which results in the ability to conform to the external structure of the human body,

70 and accurately follow the movement of the shoulder complex. Soft robots can be created in various

71 forms, with each form having its own method of power transmission, such as cable-driven devices

72 (11,12), shape-memory actuators (13), combustion (14), magnetism (15), and pressurized fluids (16).

73 Cable-driven actuators (17) most closely mimic the structure of the muscular system but require

74 accurate positioning of anchor points.

75 Another form of power transmission for soft robotics, pressurized fluids and specifically compressed 76 air, have proven to be a popular choice for driving clinical exoskeletons(18-22). However, most fluidic

77 actuators are unable to provide sufficient mobility for the shoulder. Simpson et al constructed an 78 exosuit that provides abduction support. The suit is primarily actuated by a reinforced inflatable bladder

79 placed on the armpit (23) We also constructed a similar device by placing an inflatable bladder on the

80 superior side of the humerus. Inflating the bladder increases its stiffness and generates abduction

81 support for the user (24). O'Neill et al constructed an exosuit capable of providing support for

82 abduction, and horizontal flexion and extension. While their exoskeleton also possesses two-degrees

83 of freedom (DOF), it is unclear if it is capable of performing reaching actions (e.g. forward flexion),

84 which is essential in performing a variety of ADLs (25). Moreover, evidence regarding the efficacy of

85 pneumatic shoulder exoskeletons is scarce. To the extent of the authors' knowledge, testing data on

86 humans has only involved a limited number of subjects $(23,27)$. An expanded test on a greater number

87 of human subjects would provide a more conclusive picture on the effectiveness of these exoskeletons 
88 in performing rehabilitative tasks. Moreover, it can also aid future research towards the implementation

89 of additional features into pneumatic exoskeletons.

90 This study presents a soft robotic, pneumatic, shoulder exoskeleton with 2-DOF (Figure 1). The

91 exoskeleton is capable of performing forward flexion and extension, abduction and adduction, and

92 horizontal flexion and extension. It can likewise drive the upper arm to any pose in between these

93 standard positions. At the core of the exoskeleton are four modular, pneumatic bending actuators with

94 separated and replaceable inflation modules. Two antagonistic actuator pairs are placed along the

95 length of the humerus. One side of each pair is responsible for one direction of motion while the agonist

96 drives the humerus in the opposite direction. The exoskeleton is unique in that the synchronized and

97 simultaneous operation of the antagonistic pairs, coupled with the additional flexibility offered by

98 separated modules, allows for multi-directional movement of the exoskeleton.

99 A detailed description of the design of the actuator and exoskeleton are presented. Changes in the 100 actuator's pose and output blocked torque as a function of input pressure and actuator bending angle

101 were measured. We previously presented a hybrid plastic-fabric actuator with replaceable inflation

102 modules (24). The present study introduces a superior fabric actuator that possesses the key

103 characteristics of the hybrid actuator while eliminating its most restrictive limitations. The reduction

104 in the internal mechanical resistance of the actuator and the reduced volumetric footprint brought about

105 in the new design allow an actuator pair to create three-dimensional (3-D) bends. Finally, an

106 experiment was conducted on ten healthy subjects in order to ascertain the exoskeleton's effects on the

107 shoulder's muscle activation and trajectory. In the subsequent sections, shoulder positions follow the

108 globe coordinate system (26) while a separate system is used to describe the actuator. The coordinate

109 system of the actuator enables a more intuitive understanding of its mechanics. The neutral position of

110 the arm coincides with the $-180^{\circ}$ position of the actuator, while an arm elevation of $90^{\circ}$ coincides with

111 the actuator's $-90^{\circ}$ position. 


\section{Methods}

\section{$113 \quad 3.1 \quad$ Actuator Design}

114 The primary purpose of the actuators is to provide a combination of torques to the upper arm such that

115 the exoskeleton can push the arm to any point on the anatomical workspace in one single motion. In

116 order to do so, the actuators must be able to combine their operation in that operation of multiple

117 actuator pairs can generate diagonal motion of the humerus. Moreover, any shoulder exoskeleton,

118 whether hard or soft, must not cause a significant increase in inertia and its motors' or actuators' centers

119 of rotation must always coincide with that of the humeral head in order to avoid injury. In order to

120 address these functional requirements, the actuator used in the present study is composed of a fabric

121 spine and fabric inflation modules (Figure 2-A). The use of pneumatics reduces the mass of the

122 actuators as compared to hydraulics. Flexible 3-D printed structures are placed on the spine and

123 modules to serve as mating and locking mechanisms. The fabrication steps are detailed in additional

124 file 1. The actuator is activated through the injection of compressed air, with a maximum continuous

125 applied pressure limited to $80 \mathrm{kPa}$ in order to minimize safety risks. The resulting spatial interference

126 between adjacent, inflated modules generates a bending moment that causes the actuator to generate

127 torque. The torque generated by pneumatic actuators is directly related to their size $(27,28)$. Module

128 sizing was restricted to ensure a moderate exoskeleton size that is wearable.

129 The actuator is assembled by combining appropriate modules of varying lengths and widths based on

130 mechanical requirements (Figure 2-B). A long groove is placed on the 3D-printed spine structure,

131 which mates with corresponding beams on the module structure. This pair acts as a guide so that the

132 two components are properly placed. Slots are placed on the spine structure which subsequently mate

133 with studs on the module. The studs snap into place and lock the module into position (Figure 2-C).

134 A strip of industrial-grade hook fastener is placed on the spine, which is then subsequently attached to 
135 a neoprene sheet (i.e. exoskeleton base). The modules are then secured into place, and pneumatic lines

136 are plugged into each module using polyethylene (PET) connectors. The pneumatic lines start from a

137 pressure regulator or valve and branch out to the individual modules. Fabric straps are placed along the

138 center of the modules in order to prevent unnecessary inflation along the covered area (Figure 2-B).

139 Without the straps, the middle section (i.e. section covered by the straps) will inflate but will not

140 contribute in the generation of torque and will decrease actuation speed (29). In its neutral, deflated

141 state, the adjacent modules fold up on the sides. The actuators behave as continuum structures when

142 pressurized. The folded-up modules inflate and spatially interfere with adjacent modules. When

143 unloaded and unattached to external structures, the actuator curls into a smooth spiral shape until each

144 module is in point contact with its adjacent module (Figure 2-D). The separated structure of the

145 inflation modules, the flexibility of the fabric spine, and the absence of side seams grants

146 unprecedented 3-D flexibility to the actuator. Figure 2-E shows an inflated actuator in a helical pose.

147 The helical shape is only achievable through the actuators' flexibility. This enables the actuator to

148 perform tight 3-D bends while still maintaining its torque output in its active axis of rotation. This

149 ability to perform 3-D bends allows the actuators to combine their directions of motions while the

150 modular feature of the actuators allows it to reposition its midpoint so that the exoskeleton may be

151 adjusted to each user.

\section{$152 \quad 3.2 \quad$ Actuator Principle of Operation}

153 In order to fully understand the principle of operation of the actuator, a physical model was created.

154 The actuator generates force, and subsequently torque, through the spatial interference between

155 adjacent modules. There are $n$ modules installed in each actuator, with $n / 2+1$ intersecting pairs of

156 modules. Each $j$ th pair consists of the $i$ th and $i+1$ modules. Each module pair generates its own force

$157\left(\vec{F}_{j}\right)$, depending on the nature of the intersection of the modules, as well as their interaction with the

158 environment, and subsequently contributes to the overall torque produced by the actuator. This means 
that an actuator's torque output will differ depending on the topology of the object to which it is

160 attached. This model simulates an actuator's attachment to the human shoulder and assumes that the

161 actuator is bent at the center, while the remaining modules remain straight. In their inflated state, the

162 modules can effectively be considered as an extruded ellipse (Figure 3-A, Figure 3-B) with the lengths

163 of the minor and major axes to be equal to half of the module's inflated height $\left(\mathrm{H}_{\mathrm{i}}\right)$ and length $\left(\mathrm{Li}_{\mathrm{i}}\right)$.

164 Figure 3-C shows the free-body diagrams upon which the rest of the model is based. In this

165 configuration, the actuator effectively generates torque with respect to the pivot point; it can be

166 effectively divided into two equal segments, with each segment generating equal but opposing torques

167 that effectively bring the entire system into static equilibrium. Each segment is further subdivided into

168 three sections: the angled section (middle pair, red), the edge section (first and last pair, yellow), and

169 the straight middle section (all other pairs, blue). Each section will have its own unique mechanical

170 behaviors; the subsequent subsections will concentrate on the $n / 2+1$ to the $n$th module. The total torque

171 ( $\left.T_{\text {Total }}\right)$ generated by the actuator is the sum of each module's generated torque $\left(T_{i}\right)[1]$.

172

$$
T_{\text {Total }}=\sum_{i=1}^{n / 2} T_{i}[1]
$$

\section{$174 \quad$ 3.2.1 Force from module intersections $\left(\overrightarrow{\boldsymbol{F}}_{\boldsymbol{j}}\right)$}

$175 \vec{F}_{j}$ is dependent on the nature of the contact area (Ac) between two colliding modules. Due to the 176 elliptical shape of the modules, this model approximates the contact area as a rectangle. The contact

177 area can be determined by examining the cross-section as seen from the top-view (Figure 3-D). The

178 centers, $\mathrm{C}_{\mathrm{i}}=\left(\mathrm{C}_{\mathrm{i}, \mathrm{x}}, \mathrm{C}_{\mathrm{i}, \mathrm{y}}\right)$ and $\mathrm{C}_{\mathrm{i}+1}=\left(\mathrm{C}_{\mathrm{i}+1, \mathrm{x}}, \mathrm{C}_{\mathrm{i}+1, \mathrm{y}}\right)$ of the two ellipses are first established; in the proceeding

179 calculations, both centers lie on the $\mathrm{x}$-axis while the origin coincides with $\mathrm{C}_{\mathrm{i}+1}$. Points $\mathrm{Mi}_{\mathrm{i}}=\left(\mathrm{MI}_{\mathrm{I}, \mathrm{x}}, \mathrm{M}_{\mathrm{Ii}, \mathrm{y}}\right)$

180 and $\mathrm{M}_{\mathrm{i}+1=}\left(\mathrm{M}_{\mathrm{i}+1, \mathrm{x}}, \mathrm{M}_{\mathrm{i}+1, \mathrm{y}}\right)$ are the points of attachment on the fabric spine. Upon inflation, modules 
181 collide and rotationally displace by the bending angle $\left(\theta_{\mathrm{j}}\right)$. They then trace a circular path centered 182 around point $\mathrm{O}_{\mathrm{j}}$; the arc length of this path is equal to module spacing $(\mathrm{d})$. $\mathrm{C}_{\mathrm{I}, \mathrm{x}}$ can then be calculated:

$$
C_{i x}=H_{i} \sin \frac{\theta}{2}+\frac{2 d}{\theta} \sin \frac{\theta}{2}+H_{i+1} \sin \frac{\theta}{2}
$$

$$
C_{i x}=\left(H_{i}+H_{i+1}+\frac{2 d}{\theta}\right) \sin \frac{\theta}{2}
$$

185

186

187

188

190

With the location of the centers known, the ellipses can now be mathematically defined with [4] and [5]

$$
1=\left(\frac{\left(x_{i}-C_{i x}\right)\left(\cos \frac{\theta}{2}\right)+\left(y_{i}-C_{i y}\right)\left(\sin \frac{\theta}{2}\right)}{\frac{H_{i}}{2}}\right)^{2}+\left(\frac{\left(y_{i}-C_{i y}\right)\left(\cos \frac{\theta}{2}\right)-\left(x_{i}-C_{i y}\right)\left(\sin \frac{\theta}{2}\right)}{\frac{L_{i}}{2}}\right)^{2}
$$

$$
1=\left(\frac{\left(x_{i+1}-C_{i+1 x}\right)\left(\cos \frac{\theta}{2}\right)+\left(y_{i+1}-C_{i+1 y}\right)\left(\sin \frac{\theta}{2}\right)}{\frac{H_{i+1}}{2}}\right)^{2}+\left(\frac{\left(y_{i+1}-C_{i+1 y}\right)\left(\cos \frac{\theta}{2}\right)-\left(x_{i+1}-C_{i+1 y}\right)\left(\sin _{\frac{\theta}{2}}\right)}{\frac{L_{i+1}}{2}}\right)^{2}
$$

The points of intersection, $\mathrm{I}_{\mathrm{j}, 1}=\left(\mathrm{I}_{\mathrm{j}, 1, \mathrm{x}}, \mathrm{I}_{\mathrm{j}, 1, \mathrm{y}}\right)$ and $\mathrm{I}_{\mathrm{j}, 2}=\left(\mathrm{I}_{\mathrm{j}, 2, \mathrm{x}}, \mathrm{I}_{\mathrm{j}, 2, \mathrm{y}}\right)$, between the two ellipses are determined by simultaneously solving [4] and [5]. The quadratic nature of expressions [4] and [5] can produce up to four points in intersection. To prevent the model from underestimating the size of the contact area, the two points with the largest linear distance between them are chosen. The rectangular area $A_{c}$ is then calculated using [6], where $\mathrm{W}_{\mathrm{i}}$ is the module's width. The line of action of the force vector $\left(\vec{F}_{j}\right)$ is assumed to lie within the center of and is normal to Ac; $\vec{F}_{j}$ is calculated through [7], where $\mathrm{P}$ is the pressure inside the modules. 


$$
A_{C}=d\left(I_{j, 1}, I_{j, 2}\right)\left(W_{i}\right)
$$

$$
\vec{F}_{j}=\left(P A_{C}\right) \cdot \frac{I_{j, 1} x I_{j, 2}}{|| I_{j, 1} x I_{j, 2} \mid} \text { where } \vec{F}_{j}=\left(F_{j, x}, F_{j, y}\right)
$$

\subsubsection{The angled section $\left(\frac{n}{2}+1\right.$ and $\frac{n}{2}-1$ module $)$}

The angled section transmits a vertical force and a horizontal force to the arm or body which is subsequently converted to torque. The vertical force is effectively transmitted as a linear distributed load, while the horizontal force can be effectively considered as a point load. This horizontal force is also opposed by the contact in the $j / 2+1$ pair; it is important to note that the modules of the $j / 2+1$ pair are oriented $0^{\circ}$ from each other (i.e. $\left.\theta=0^{\circ}\right)$. The torque generated by the angled section $\left(T_{\frac{n}{2}+1}\right)$ is

210 given by [8].

211

$$
T_{\frac{n}{2}+1}=H_{\frac{n}{2}} x\left(\vec{F}_{\frac{n}{4}}-\vec{F}_{\frac{n}{4}+1}\right)+\frac{d\left(M_{\frac{n}{2}+1}-I_{\frac{n}{4}}, 1\right)}{3} x\left(\vec{F}_{\frac{n}{4}}\right)
$$

212 At $\theta>-90^{\circ}$, the vertical component of $\vec{F}_{j}\left(\vec{F}_{j y}\right)$ no longer contributes to torque generation due to the

213 fact that vertical force is now directed away from the arm, and is transformed into tension on the

214 fabric spine, as opposed to generating a counter-acting force on the arm. This results in minor

215 compression of the actuator but does not significantly affect its performance. At $\theta>-90^{\circ}, T_{\frac{n}{2}+1}$ is

216 described by [9].

$$
\boldsymbol{T}_{\frac{n}{2}+1}=H_{\frac{n}{2}+1} x\left(\vec{F}_{\frac{n}{4}}-\vec{F}_{\frac{n}{4}+1}\right)
$$




\subsubsection{The middle section (all other modules)}

219 It can be seen in Figure 3-C that each module along the middle sections generates equal and opposing

220 forces from their intersection with their neighboring modules. This results in zero torque generation

221 for modules in the middle section.

223

222

$$
T_{i}=H_{i} x\left(\vec{F}_{j}-\vec{F}_{j+1}\right)=0 \text { where } n \neq 1, n, \frac{n}{2}+1, \frac{n}{2}-1 \text { and } j \neq \frac{n}{2}, \frac{n}{4}, \frac{n}{4}-1[10]
$$

\subsubsection{The edge section (1st and nth module)}

225 It is assumed that the modules adjacent to the edge module (i.e. 2 nd and n-1 module) are oriented

226 parallel to the edge module (i.e. $\theta=0^{\circ}$ ). Therefore, only a single force generates torque for the

227 module. The torque generated by the edge section $\left(T_{n}\right)$ is given by [11]

$$
T_{n}=H_{n} x\left(\vec{F}_{\frac{n}{2}}\right)
$$

230 Finally, (1) can be expanded into (12)

$231 T_{\text {Total }}=\left\{\begin{array}{r}H_{\frac{n}{2}+1} x\left(\vec{F}_{\frac{n}{4}}-\vec{F}_{\frac{n}{4}+1}\right)+\frac{d\left(M_{\frac{n}{2}+1}-I_{\frac{n}{4}}\right)}{3} x\left(\vec{F}_{\frac{n}{4}}\right)+H_{n} x\left(\vec{F}_{\frac{n}{2}}\right), \theta_{\frac{n}{4}} \leq-90 \\ H_{\frac{n}{2}+1} x\left(\vec{F}_{\frac{n}{4}}-\vec{F}_{\frac{n}{4}+1}\right)+H_{n} x\left(\vec{F}_{\frac{n}{2}}\right), \theta_{\frac{n}{4}}>-90\end{array}\right.$

\subsection{The 2-DOF Exoskeleton}

233 The base of the exoskeleton is a customized neoprene sleeve that acts as a platform to which the spines

234 can be attached. The neoprene sleeve features straps along the torso to ensure that it remains secure 
each actuator positioned $90^{\circ}$ from each other (Figure 1). Each pair is responsible for each degree of

237 freedom - one pair performs humerus elevation and depression, while another performs rotation of the

238 humerus' plane of elevation. The depression actuator starts from the base of the neck, tracing the

239 superior side of the humerus. The elevation actuator is placed on the inferior side, starting from the

240 lateral area of the ribcage and extending through the armpit and the arm. The steering actuators are

241 placed on the anterior and posterior arm starting from the sternum and the spine, respectively.

242 Additional modules, positioned as to have minimum possible contact area, are added along the upper

243 arm. These modules have minute overlap and are essentially in point contact when pressurized. They

244 act as a means to distribute the load generated by the bending actuators throughout the entire upper

245 arm. These modules slightly bend and effectively act as straight beams. Such a seamless system could

246 easily be extended to other joints in the arm, such as the elbow and the wrist. However, a system for

247 the entire arm is beyond the scope of this paper. The exoskeleton is controlled by a pneumatic control

248 system; exoskeleton output is controlled by regulating the pressure supplied to the actuators. The

249 details of the control system are available in additional file $\mathbf{1 .}$

250 The operation of the actuators allows the humerus to trace a natural trajectory. From the neutral

251 position, the humerus can perform shoulder abduction and adduction by activating the elevation or

252 depression actuators (Figure 4-A). The shoulder can also be rotated along the plane of elevation by

253 sequentially activating the elevation and steering actuators (Figure 4-B). Simultaneous activation of

254 the elevation and steering actuators also grants the ability to perform shoulder flexion or extension

255 (Figure 4-C). A video of these movements is made available to supplement this paper (Additional file

256 2). In addition to performing the basic anatomical movements, the controlled activation of both steering

257 actuators and the elevation actuators allows the performance of reaching actions (Figure 4-D, Figure 258 4-E). 
260 In order to verify the accuracy of the physical model, three actuator variants were constructed, with

261 each variant having a total of eight modules installed. The variants differ in the size and pattern of the

262 modules installed. Their geometric parameters are listed in Table 1. Module widths were based on the

263 lower 5th percentile of female upper arm diameters for B, and the lower 5th percentile of male upper

264 arm diameters for C (27). Module spacing (d) was set at $25 \mathrm{~mm}$ in order to minimize the discontinuity

265 in the curvature profile of the actuator. While tighter spacing is desired, the size of the 3-D printed

266 locking structures presents a practical minimum. Alternate module patterning (i.e. ABAB, ACAC) was

267 chosen since a homogenous pattern induced buckling in preliminary tests. This pattern involves the

268 installation of alternating module sizes; specifically, module A was installed between each B and C

269 module. The module lengths were then set to $65 \mathrm{~mm}$ and $90 \mathrm{~mm}$ to ensure sufficient spatial interference

270 between adjacent modules.

271 The actuator' static free bending output in response to a pressure input was measured by hanging the 272 actuator vertically. The detailed setup is available in additional file $\mathbf{1}$.

TABLE1. MODUle Dimensions AND

PATTERNING

\begin{tabular}{|c|c|c|}
\hline $\begin{array}{c}\text { Module } \\
\text { Variants }\end{array}$ & $\begin{array}{c}\text { Module } \\
\text { Length }(\boldsymbol{L})\end{array}$ & $\begin{array}{c}\text { Module } \\
\text { Width }(\boldsymbol{W})\end{array}$ \\
\hline A & $65 \mathrm{~mm}$ & $55 \mathrm{~mm}$ \\
\hline B & $90 \mathrm{~mm}$ & $55 \mathrm{~mm}$ \\
\hline C & $90 \mathrm{~mm}$ & $65 \mathrm{~mm}$ \\
\hline $\begin{array}{c}\text { Actuator } \\
\text { Variant }\end{array}$ & Module Patterninga \\
\hline D1 & \multicolumn{2}{|c|}{ AAAAAAAA } \\
\hline D2 & \multicolumn{2}{|c|}{ ABABABAB } \\
\hline D3 & \multicolumn{2}{|c|}{ ACACACAC } \\
\hline
\end{tabular}

a. The installation arrangement of modules 
273 A two-axis torque measurement system was used to measure the actuators' static performance (Figure

274 5-A). As an inflatable structure, an inflated actuator will always attempt to remain in a 2-D pose by 275 generating a straightening torque when acted upon by an external force that drives it into a 3-D pose.

276 While the actuator is only capable of generating torque along its primary axis, it is important to quantify

277 how much unwanted straightening torque the actuators generate when forced into a 3-D pose. As shown

278 in Figure 5-B and Figure 5-C, the A-A' axis coincides with the actuator's primary axis of bending,

279 while the B-B' axis is perpendicular to A-A' axis. The A-A' angle corresponds to $\theta$ in the physical 280 model. A fully folded actuator corresponds to an A-A' angle of $-180^{\circ}$ while $0^{\circ}$ is straight. A detailed 281 explanation of the measurement system is available in additional file $\mathbf{1}$.

282 All experiments were repeated three times, and the samples were dismounted and remounted before 283 each repetition in order to minimize the effects of any actuator movement that may have occurred 284 during a measurement.

\subsection{Healthy Subject Testing Protocol}

Ten healthy subjects were recruited into the study; the test was reviewed and approved by NUS Institutional Review Board (N-17-103). Willing subjects who had no history of injuries relating to the upper limb and possess body mass index (BMI) between the range of 18.5 to 24.9 were allowed to participate. A summarized list of the recruited subjects' anthropomorphic measurements related to the upper arm is available in Additional File 1. Subjects were only required to attend a single session. Informed consent was acquired at the start of the session and anthropomorphic measurements were recorded.

The exoskeleton was adapted to the subject at the start of the test by changing the modules attached. Actuator variant D2 was attached and the number and spacing of non-bending modules were adjusted until the length of the actuators reached the elbow. After acquiring consent, surface electromyography 
(sEMG) sensors (Delsys, Trigno Wireless) were attached to the lateral, anterior and posterior deltoid,

297 the clavicular head of the pectoralis major and the infraspinatus relating to the right arm. The test 298 focused on the right arm of the subject in order to circumvent electrocardiograph (ECG) contamination

299 of the sEMG signals. Meanwhile, an inertial measurement unit, or IMU, (Bosch, BN055) was attached

300 to the chest, lying on the sternum and oriented parallel to the transverse plane; another IMU was

301 attached to the medial right, upper arm. While motion capture technology is typically used for such

302 tests [118], [133]-[136], this technique relies on the application of passive motion markers on the limb

303 that are tracked by optical cameras. The nature of the exoskeleton prohibits the utilization of motion

304 capture due to the fact that the inflatable modules have the tendency to block the line of sight of the

305 camera to the markers. The IMUs are positioned in a manner wherein they are in identical orientations

306 when the arm is abducted to $90^{\circ}$. Figure 6 shows the application of the sEMG and IMU sensors. The

307 sEMGs and IMUs were sampled at $2000 \mathrm{~Hz}$ and $100 \mathrm{~Hz}$ respectively. To facilitate IMU mapping during

308 data processing, subjects were instructed to position their arm into three standard anatomical positions:

309 the neutral position, $90^{\circ}$ shoulder abduction, and $90^{\circ}$ forward flexion (Figure 6-D). IMU readings were

310 then recorded as the arms were kept stationary at these positions. Warm-up exercises were performed

311 for approximately five minutes. At this stage, the subjects were also asked to familiarize themselves

312 with the associated movements involved in the test. Maximum voluntary contraction (MVC) of the

313 muscles was recorded next. Subjects were asked to place their limbs in the neutral position; a researcher

314 then held the arm stationary while the subject attempted to perform shoulder abduction; a similar action

315 activity was performed for shoulder flexion. From the $90^{\circ}$ abduction position, subjects performed

316 horizontal shoulder flexion.

317 Three arm motions were investigated: shoulder abduction and adduction, shoulder flexion and 318 extension, and shoulder horizontal flexion and extension. Movements were performed until the arm

319 reached a minimum of $90^{\circ}$ of the target movement, and the reverse motion was subsequently 
320 performed. Each movement was repeated three times. Each type of motion was conducted under

321 different speeds - fast and slow. The slow speed corresponds to an angular velocity of $30^{\circ} / \mathrm{s}$ while fast 322 movements corresponded to an angular velocity of $90 \%$ s. These values correspond to the average

323 execution speed of ADLs involving the human shoulder (30). Subjects guided themselves during

324 movements. At the peak of each movement, the subject was instructed to pause in order to ensure that

325 each movement was isolated from the proceeding movement. Once the sensors were applied, the

326 subjects donned a neoprene sleeve to ensure that the sEMG sensors remain stationary and to minimize

327 the presence of motion artifacts.

328 The test was conducted in three phases. The first phase (free phase, F) serves as a baseline; the subjects

329 were asked to perform arm motions while only wearing an empty neoprene sleeve (Figure 6-E). In the

330 second phase (unpowered phase ,U), the neoprene sleeve was removed, and the subjects wore an

331 unpowered exoskeleton (Figure 6-F). At this stage, the exoskeleton was disconnected from the control

332 system. Subjects continued to perform arm motions under their own power. This phase is designed to

333 measure the mechanical resistance of the exoskeleton in terms of the amount of muscular effort

334 necessary to move the arm and the exoskeleton. Finally, the exoskeleton was powered in the third phase

335 (powered phase, P). During the powered phase of horizontal flexion and extension, subjects elevated

336 their arm under their own power.

337 Data sets were segmented based on the condition (i.e. free, unpowered, or powered), and movement

338 direction. Comparisons were performed by calculating the root mean square (RMS) of the IMU 339 trajectory data and normalized muscle activation data for each segment. Overall, three comparison 340 types were made based on their conditions: powered against free $(\mathrm{PvF})$, powered against unpowered $341(\mathrm{PvU})$, and unpowered against free $(\mathrm{UvF})$. The data processing techniques are detailed in Additional $342 \quad$ File 1. 


\section{Results}

\section{$344 \quad 4.1 \quad$ Actuator Static Characteristics}

345 Each actuator, when unloaded, was able to achieve full bending $\left(>360^{\circ}\right)$ when excited with a pressure

346 of 10kPa. An increase in pressure did not have any effect on its pose.

347 Figure 7-A shows the results of each variant's torque output, as a function of A-A' angle, at $30^{\circ}$

348 increments. The measurements were acquired by setting the $\mathrm{B}-\mathrm{B}^{\prime}$ angle to $0^{\circ}$ and pressurizing the 349 actuator to $80 \mathrm{kPa}$. Meanwhile, Figure 7-B shows the torque output of the actuator as a function of the 350 input pressure. These measurements were taken at an A-A' angle of $-90^{\circ}$ and a B-B' angle of $0^{\circ}$. The 351 pressure inside the actuators was varied from $0 \mathrm{kPa}$ to $80 \mathrm{kPa}$. It can be seen that the static physical 352 model is able to accurately follow the behavior of the actuators as observed by the experiments. When 353 predicting the relationship between torque and angle, as seen in Figure 7-A, the model had mean errors 354 of $21.7 \%, 20.9 \%$, and $15.8 \%$ for D1, D2 and D3 variants, respectively. The model exhibited mean 355 errors of $16.2 \%, 28.3 \%$ and $14.9 \%$ when predicting torque verses pressure response (Figure 7-B). 356 Overall, both the data and the model suggest that the actuator exhibits an exponential decrease in torque 357 output as the A-A angle is increased. The actuators are able to apply maximum torque from A-A' angle $358-180^{\circ}$ to $-90^{\circ}$, with variants D1, D2 and D3 exhibiting peak torques of $10.24 \mathrm{~N}-\mathrm{m}, 11.15 \mathrm{~N}-\mathrm{m}$ and $35915.54 \mathrm{~N}-\mathrm{m}$, respectively. Meanwhile, they exhibit torques of 1.27 N-m, $4.44 \mathrm{~N}-\mathrm{m}$, and 4.66 N-m at $36090^{\circ}$, the angle at which the arm imposes maximum static load on the actuator $\left(-90^{\circ} \mathrm{A}-\mathrm{A}^{\prime}\right.$ angle 361 corresponds to $90^{\circ}$ of shoulder elevation). The actuator's torque output effectively becomes constant 362 at $\mathrm{A}-\mathrm{A}^{\prime}$ angle $0^{\circ}$ to $90^{\circ}$. They exhibited torques of $0.84 \mathrm{~N}-\mathrm{m}, 1.54 \mathrm{~N}-\mathrm{m}$, and $1.80 \mathrm{~N}-\mathrm{m}$ at on this range.

363 The unfolding nature of the modules ensure that the contact area is nearly constant when the actuator 364 is positioned between $0^{\circ}$ to $90^{\circ}$. They also suggest that torque output has a linear response to increases 365 in pressure. 
366 An increased reduction in available contact area occurs when the A-A' angle and B-B' angle change

367 simultaneously. The effects of this behavior on variant D2 are shown in Figure 7-C. In this 368 measurement, the $\mathrm{A}-\mathrm{A}^{\prime}$ angle was set to $-90^{\circ}$ and the $\mathrm{B}-\mathrm{B}^{\prime}$ angle was varied from $45^{\circ}$ to $0^{\circ}$ at $15^{\circ}$

369 increments. This range of motion corresponds to that required of the majority of ADLs (19). The

370 actuator was then pressurized to $80 \mathrm{kPa}$. An overall decrease in the torque output along the A-A' axis

371 was found as the B-B' angle was increased. However, the platform was not able to detect any

372 significant torque output $(>0.5 \mathrm{~N}-\mathrm{m})$ along the B-B' axis. This can be attributed to the ability of each

373 individual module to translate in 3-D as well as the aspect ratio of the modules; they show minimal

374 surface area along the B-B' axis. Effectively, the modules reposition themselves such that there is

375 minimal resistance during operation, resulting in small B-B' torques.

\section{$376 \quad 4.2 \quad$ Arm Trajectory}

377 Trajectories extracted from a single subject are shown in Figure 8. Each subject was able to reach the

$37890^{\circ}$ arm position for all motions as observed during the test. Application of the exoskeleton increased

379 the starting angle of elevation from $0^{\circ}$ (i.e. neutral position/pendulum position) to $\sim 15$. However, it is

380 interesting to note that each subject was able to return to true neutral position (i.e. $0^{\circ}$ ) after the first

381 cycle. This is attributed to the forced movement of the subject, allowing each subject to overcome the

382 resistance introduced by the exoskeleton and forcibly return to true neutral. A closer visual inspection

383 of the individual trajectories shows an observable difference in the paths among the three phases.

384 The full tabular data of the change in RMS of the arm trajectories are found in Additional file 1 (Table

385 S2 and Table S3). Fast, arm elevation movements (i.e. fast abduction and fast forward flexion) show

386 a definite change in arm trajectory in PvF comparisons. However, the change in trajectory across

387 subjects is not consistent, with most results being statistically insignificant (i.e. $p>0.05$ ) in PvF and

388 PvU comparisons. No statistically significant results were observed for UvF comparisons which can 
suggest that the mechanical structure itself does not cause this change in trajectory. Instead, the change

390 in the behavior of arm movements is attributed to the dissimilar movement of the actuator and the arm,

391 and not due to the mechanical resistance introduced by the exoskeleton. It's also interesting to note

392 that the exoskeleton does not affect horizontal flexion and extension; while there was an $8 \%$ change in

393 the trajectory $\mathrm{PvU}$ horizontal extension, this change is minimal.

\subsection{Muscular Activation}

395 Figure 8 also features the time-series muscle activation — shown as EMG voltage readings normalized 396 to MVC - of a single subject across various movements and phases. Visual analysis indicates a 397 correlation between muscle activation and arm position: muscle activation increases as arm is flexed 398 or abducted and respectively decreases as it is extended or adducted. These results are expected given 399 that the mechanical load on the muscles increases as the arm is positioned away from the neutral 400 position. Among the same the movement and muscles, the shapes of the activation graphs show similar 401 patterns and structure. However, there is an apparent offset between the plots from different phases 402 suggesting a change in the overall magnitude of muscle activation between the free, unpowered, and 403 powered phases. Figure 9 and figure 10 show the summarized relative change in muscle activation for 404 relevant muscles for slow and fast movements respectively. Numerical versions of these results are 405 provided in Additional file 1 (Table S4 and Table S5).

406 The results show a decrease in the mean RMS of muscle activation in the population for fast PvF 407 comparisons. Statistically significant results were obtained from multiple muscles during fast 408 exercises: the lateral deltoid and infraspinatus during abduction and adduction, the anterior deltoid 409 during forward flexion and extension, the pectoralis major during horizontal flexion, and the pectoralis 410 major and posterior deltoid during horizontal extension. The exoskeleton was able to reduce deltoid

411 activation by $65 \%(\mathrm{p}<0.01)$ when performing abduction and adduction, and by $45 \%(\mathrm{p}<0.01)$ and $25 \%$ 
$412(\mathrm{p}<0.05)$ when performing forward flexion and extension, respectively. The activation of the pectoralis

413 major was reduced by $34 \%(\mathrm{p}<0.05)$ when rotating the arm's angle of plane of elevation. While

414 reductions in some muscles were also observed during slow movements, the reduction magnitude was

415 considerably lower as compared to those acquired during fast movements. Statistically significant

416 results were only obtained for the anterior deltoid and lateral deltoid during slow forward flexion and

417 extension, and the anterior deltoid for horizontal extension. The slow movements only apply minimal

418 loads to the shoulder muscles that result in low magnitudes in the measured EMG voltages, which

419 explains the high variance in non-significant findings. The assistance provided by the exoskeleton

420 would not be apparent in the voltage waveform due to the inherent, relatively low signal-to-noise ratio

421 of EMG (31). Nevertheless, the statistically significant results for forward flexion and extension

422 indicate that the exoskeleton is capable of providing support during reaching movements.

423 Similar to PvF comparisons, PvU comparisons showed reductions in muscle activations. Statistically

424 significant results were obtained during fast movements for all relevant muscles in abduction and 425 adduction, horizontal flexion and extension, and the anterior deltoid for forward flexion and extension.

426 These particular muscles exhibited similar results in PvF comparisons. Meanwhile slow movements

427 yielded more statistically significant values in contrast to PvF. All muscles relating to abduction and

428 adduction, the pectoralis major during horizontal flexion and extension, and the lateral deltoid during

429 forward flexion and extension also achieved statistical significance.

430 Meanwhile, the results for UvF comparisons vary for both speeds. Pectoralis major activation increased 431 by $29 \%(\mathrm{p}<0.05)$ while performing fast horizontal flexion and extension. Lateral deltoid activation 432 increased by $25 \%(\mathrm{p}>0.05)$ and $28 \%(\mathrm{p}>0.05)$ while performing slow abduction and adduction, 433 respectively, while infraspinatus activation increased by $10 \%(\mathrm{p}>0.05)$ when performing slow 434 abduction and adduction. Other muscles did not feature statistically significant readings. Most notably, 435 forward flexion and extension did not exhibit any change in muscle activation for both speeds. The 
436 high variance in the data is attributed to the various strategies employed by the subjects in order to defy

437 the mechanical resistance added by the exoskeleton, with some strategies more successful than others.

438 Moreover, the fit of the sleeve to a participant may also play a role in the amount of mechanical

439 resistance it would impart on the user.

4405 Discussion

441 The ultimate goal of this paper is to create an effective and elegant solution to the current mechanical

442 and kinematic complexities surrounding the design of robotic shoulder exoskeletons for clinical

443 rehabilitation. To avoid the complexities involved in using traditional robotic architectures, we

444 employed a fabric-based, pneumatic soft actuator to drive a shoulder exoskeleton. The actuator was

445 developed based on the hybrid fabric-plastic design and results we previously presented $(27,28)$. The

446 majority of the actuator is composed of nylon fabric, which resulted in negligible bending resistance

447 as evidenced by its ability to trace its full range of motion with minimal input. This feature, along with

448 its soft robotic nature, ensured that the shoulder sleeve will have maximum mechanical transparency.

449 Results from various tests indicate that the exoskeleton will be able to provide torque assistance

450 regardless of the position of the actuators or the pressure supplied. Nevertheless, the positions of the

451 actuators influence their torque outputs. Load bearing actuators must operate from the $-180^{\circ}$ to $-90^{\circ} \mathrm{A}-$

452 A' angle range in order to maximize their utility. This behavior is consistent with that from our previous

453 work(27,28). These actuators would supply approximately $7 \%, 24.6 \%$ and $25.8 \%$ of the torque

454 necessary to maintain arm elevation of $90^{\circ}$ for a typical, stretched, male arm with a mass of $3.5 \mathrm{~kg}(32)$.

455 Users can still benefit from the high peak torques at low elevation angles (i.e. $-180^{\circ}$ to $-90^{\circ}$ A-A') when

456 performing high velocity, dynamic movements.

457 The final design of the exoskeleton is intended to capitalize on the capabilities of the modular actuators.

458 When comparing the torque curves of the D2 and D3 variants, it can be seen that the difference is 
negligible. This suggests that minor buckling occurs with the D3 actuator and that a significant portion

460 of the energy injected in the D3 variant is transformed into unwanted curling of the actuator between

461 modules, which results in extremely diminished torque output. While this amount of buckling will not

462 pose a safety risk, the D3 variant is not suitable for use in an exoskeleton. Using hook-and-loop

463 fasteners, the actuators can be specifically placed on the body of each user. The use of neoprene as the

464 primary material for the sleeve provided the exoskeleton with an elastic base that tightly follows the

465 contours of the body. This allows the sleeve and the actuators to remain in the correct position. The

466 positioning of the actuators takes advantage of their torque-angle curve. Using this configuration, the

467 majority of the loads are to be handled by the elevation actuator. This actuator primarily operates in

468 the $-180^{\circ}$ to $-90^{\circ} \mathrm{A}-\mathrm{A}^{\prime}$ region, which is the region of maximum torque. The steering actuators reside

469 in the $0^{\circ}$ to $90^{\circ} \mathrm{A}-\mathrm{A}^{\prime}$ region, where the torque-angle relationship is effectively constant. Moreover, the

470 actuators are mounted in a parallel configuration. This allows the exoskeleton to distribute its force

471 application throughout the entire surface area of the arm. The use of antagonistic actuators also negates

472 the effect of drift during deflation since the agonist will be primarily responsible for deflating the

473 actuator. An additional feature of the modular spine is that the torque output of both pairs can be

474 combined such that they can trace trajectories outside a single actuator's axis of rotation. This can be

475 achieved through the simultaneous activation of both pairs.

476 A proper evaluation of the exoskeleton, along with the actuators, involves testing on a human shoulder

477 in order to quantify their benefits and drawbacks as well as providing insight on potential design

478 improvements that otherwise may not become apparent. The test performed for this paper examined

479 the ability of the exoskeleton to assist upper arm movement through the quantitative measurements of

480 relevant muscle activations. The comparison of powered and unpowered movements conclusively

481 demonstrated lessened muscle activation when assisted by the powered exoskeleton, which

482 consequently indicates that the exoskeleton effectively provides torque to the human arm. Likewise, 
483 the comparison of powered movements and free movements show the exoskeleton's capability to

484 reduce muscle activation. It can reduce deltoid activation by up to $65 \%$ while performing abduction 485 and adduction, and is also capable of providing multi-DOF support, which allows subjects to perform 486 forward flexion and extension. While the exoskeleton also reduced muscle activation by $45 \%$ during 487 forward flexion movements, the amount of assistance was reduced to $25 \%$ when performing the 488 equivalent unloading motion (i.e. forward extension). This is due to the reduced overall load on the 489 muscles when performing extension movements as compared to performing flexion movements. 490 Furthermore, the exoskeleton was able to steer the arm's angle of plane of elevation, which reduced 491 muscle activation by $33 \%$ when performing horizontal flexion and extension. This amount of mobility 492 is attributed to the unique actuator design. While the actuator can only actively generate torque in one 493 axis, the separated module design allows it to perform 3-D movements when acted upon by an external 494 force (i.e. a secondary actuator mounted perpendicularly). A traditional, planar, pneumatic bending 495 actuator would generate resistive torque, forcing it to return to a 2-D, planar shape, and reduce its 496 effectiveness in providing 2-DOF support.

497 The use of fabric components allowed for the construction of a powered exoskeleton that imparts 498 minimal mechanical resistance to the human arm. The fabric actuators allow for a simple exoskeleton 499 design that accurately follows the movement of the humeral head without the need for simplistic 500 biomechanical approximations or overly-complicated alignment compensation mechanisms typically 501 employed in traditional, shoulder exoskeletons(33). No unintended loading on other muscles were 502 detected during all of the tests. UvF comparisons yielded statistically significant data for horizontal 503 flexion and extension, and slow abduction and adduction, which indicate that the mechanical structure 504 of the exoskeleton increases muscle activation. While the exoskeleton may have increased the 505 mechanical resistance in other areas of the arm, this amount of resistance is presumably relatively 506 insignificant in relation to the gravity load of the arm itself and was even undetectable through sEMG. 
The mean RMS values show that activation of shoulder muscles during fast unpowered movements

508 increased by up to $29 \%$, as compared to free movements, and slow movements showed an increase of

509 up to $48 \%$. However, it must be noted that the absolute change in activation is negligible since the

510 overall load on the arm is similarly smaller during slow movements. Nevertheless, both PvF and PvU

511 comparisons yielded the same amount of muscle activation reduction, indicating that the exoskeleton

512 is not only capable of overcoming its own mechanical resistance, but also capable of effectively

513 negating it.

514 The unique soft structure of the exoskeleton also allowed subjects to retain their shoulder's natural

515 range of motion regardless of the prevailing condition (i.e. free, unpowered or powered phase). While

516 their relaxed, neutral position was elevated by approximately $15 \mathrm{o}$ when wearing the exoskeleton, each

517 subject was still able to reach the true neutral position (i.e. 0 o elevation) when effort was applied. While

518 this required the application of force on the arm — either by the user, or the exoskeleton - the results

519 show that the exoskeleton was able to compensate, and overall muscular activation was still reduced.

520 Meanwhile, the statistical results for fast forward flexion and extension, fast abduction, and slow

521 abduction seem to suggest that use of the exoskeleton affects elevation movements. These nevertheless

522 indicate that the use of the exoskeleton, in its current form, will reduce the kinematic accuracy of the

523 user's arm. It is important to note that subjects were only given one day to use the exoskeleton, and

524 further training and familiarization may allow them to regain movement accuracy (34) .

525 The results highlight the positive benefits of the exoskeleton to the user by measuring sEMG signals 526 as way to ascertain its effects on muscle activation. A comprehensive comparison of the results of the 527 exoskeleton with that found in the literature is currently not possible due to a lack of a standard

528 evaluation protocol. By using an unsuited state as a baseline, we were able to remove the mechanical

529 resistance imparted by the actuators from our measurements. This is not always the case when

530 exoskeletons are evaluated. Nevertheless, sEMG measurements have also been used to assess 
exoskeleton performance. O'Neill et al was able to achieve $62.75 \%$ of muscle reduction on the lateral

532 deltoid when performing abduction and $16.94 \%$ on the pectoralis major when performing horizontal

533 flexion (35). Simpson et al also collected sEMG signals, but their data processing method was different

534 from the one presented here (23). Tiseni et al achieved a mean reduction of $56.9 \%$ on the lateral deltoid

535 using a hybrid soft-hard exoskeleton on five healthy subjects (36). The vast other majority of functional

536 tests with exoskeletons directly deal with subjects with shoulder impairments. They typically

537 implement clinical tests which are designed to gauge the level of shoulder functionality.

538 It is important to note that the exoskeleton lacks a controller that involves human input in the activation

539 of the exoskeleton's functions. Admittance control has proven to be effective in predicting the onset of

540 user motion and has been implemented in a number of exoskeletons $(37,38)$. Continuum-based

541 actuators impart forces on a large surface area, making it impractical to measure forces through

542 conventional sensors. While the addition of an admittance controller would improve the performance

543 of the exoskeleton, it is difficult to implement. We believe that the observed changes in arm trajectory

544 when wearing the powered exoskeleton is also a product of a lack of user input in the control system.

545 The inclusion of intent sensing would allow the exoskeleton to make on-the-fly adjustments to its

546 motion and will therefore reduce its effect on the user's kinematics. These changes are therefore a

547 product of the lack of synchronization between the user and the exoskeleton, and not of the mechanical

548 design of the exoskeleton. The build quality of the exoskeleton in the current study also limited possible

549 performance. The exoskeleton was only capable of withstanding $80 \mathrm{kPa}$ of continuous, sustained

550 pressure and $150 \mathrm{kPa}$ of intermittent pressure. Improving the build quality delays, the onset of device

551 failure, which could lead to the ability to handle higher intermittent pressures and the generation of

552 stronger torques. While there is evidence of mechanical resistance throughout the exoskeleton's range

553 of motion, the resistance of the steering actuators must be significantly reduced. Natural, free, shoulder

554 movement in this direction only presents minimal load to the shoulder muscles and the exoskeleton 
555 should therefore present the relatively same amount of low resistance. Reduced mechanical resistance

556 would also simplify the application of an admittance-based controller for backdrivability.

\section{$557 \quad 6 \quad$ Conclusion}

558 The exoskeleton presented demonstrated the feasibility of creating a fabric-based shoulder

559 exoskeleton, powered by flexible pneumatic actuators. The torque response and free bending response

560 of the underlying actuator were explored. The exoskeleton was tested on healthy subjects in order to

561 measure its efficacy. Our results quantitatively demonstrated the efficacy of our exoskeleton in

562 imparting torque to the shoulder joint. While there are limitations to our study, the results suggest that

563 the unique exoskeleton configuration is effective in providing support to the human shoulder

564 throughout its entire range of motion. We were able to create an exoskeleton configuration with two

565 degrees-of-freedom. It is able to support not only abduction and adduction, and horizontal flexion and

566 extension, but also reaching motions (i.e. forward flexion and extension). Reaching movements allow

567 the exoskeleton to assist the users in a wide variety of ADLs in a seamless manner. While these ADLs

568 may possibly be accomplished using sequential humeral elevation and rotation of the plane of

569 elevation, this series of motions is undoubtedly unnatural. Moreover, the exoskeleton was able to

570 provide support throughout the entire range of motion of the shoulder and this mainly attributed to the

571 minimal mechanical resistance of the actuator coupled with the parallel actuation configuration of the 572 exoskeleton.

\section{$\begin{array}{lll}573 & 7 & \text { List of Abbreviations and Symbols }\end{array}$}

FULL MEANING

Three-Dimensional

Activities of Daily Living

Glenohumeral
ABBREVIATION

3-D

ADL

$\mathrm{GH}$ 
Degrees-of-Freedom DOF

$\begin{array}{ll}\text { Polyethylene } & \text { PET }\end{array}$

Body Mass Index $\quad$ BMI

Actuator Active Axis of Bending A-A'

Actuator Passive Axis of Bending B-B'

$\begin{array}{ll}\text { Electrocardiography } & \text { ECG }\end{array}$

Surface Electromyography sEMG

Inertial Measurement Unit $\quad$ IMU

Maximum Voluntary Contraction $\quad$ MVC

Root Mean Square $\quad$ RMS

Powered against Free Comparison $\quad$ PvF

Powered against Unpowered Comparison $\quad$ PvU

Unpowered against Free Comparison UvF

Mathematical Interpretation Symbol

Force Generated by A Module Pair

Module Inflated Height $\quad \mathrm{Hi}_{\mathrm{i}}$

Module Inflated Length $\quad \mathrm{Li}$

$\begin{array}{ll}\text { Torque Generated by A Segment or Actuator } & T_{x}\end{array}$

Contact Area of a Module Pair $\quad$ Ac

$\begin{array}{ll}\text { Module Centroid } & \mathrm{Ci}_{\mathrm{i}}\end{array}$

Module Mounting Coordinate $\quad$ Mi

Module Bending Angle. Equivalent To A-A’ Angle $\quad \Theta_{j}$

Center Of Rotation of a Module Pair $\quad \mathrm{O}_{\mathrm{j}}$

Point of Intersection Between Adjacent Modules $\quad \mathrm{I}_{\mathrm{j}, \mathrm{n}}$

$\begin{array}{ll}\text { Applied Pressure } & \text { P }\end{array}$ 
576 The human subject test was approved by the National University of Singapore Institutional Review

577 Board (N-17-103).

5789 Consent for Publication

579 The subjects' informed consent was acquired in accordance with NUSB-IRB guidelines. Subjects

580 also consented to the publication of collected data.

$581 \quad 10 \quad$ Availability of data and materials

582 The datasets used and/or analysed during the current study are available from the corresponding

583 author on reasonable request.

\section{Competing Interests}

585 The authors declare that the research was conducted in the absence of any commercial or financial

586 relationships that could be construed as a potential conflict of interest.

\section{Author Contributions}

588 RF and RY conceptualized the project. RF manufactured the devices. RF and TMJ performed the 589 experiments. RF analyzed the results. RY provided technical guidance for the project. All authors 590 helped in editing the manuscript.

\section{$591 \quad 13 \quad$ Funding}

592 Research supported by MOE AcRF Tier 2 (R-397-000-281-112)

\section{Acknowledgements}

594 Ms. Marie Angela Ordonez for helping prepare the manuscript. 


\section{References}

596 1. Magermans DJ, Chadwick EKJ, Veeger HEJ, Van Der Helm FCT. Requirements for upper extremity motions during activities of daily living. Clin Biomech. 2005;20(6):591-9.

598 2. Terry GC, Chopp TM. Functional Anatomy of the Shoulder. J Athl Train. 2000;35(3):248-55.

3. Norouzi-Gheidari N, Archambault PS, Fung J. Effects of robot-assisted therapy on stroke

600

601

602

603

604

605

606

607

608

609

610

611

612

613

614

615

616

617

618

619

620

621

622

623

624

625

626

627

628

629 rehabilitation in upper limbs: Systematic review and meta-analysis of the literature. J Rehabil Res Dev. 2012;49(4):479-96.

4. Lum PPS, Burgar CCG, Shor PC, Majmundar M, Van der Loos M, Gresham G, et al. RobotAssisted Movement Training Compared With Conventional Therapy Techniques for the Rehabilitation of Upper-Limb Motor Function After Stroke Peter. 2002 Jul [cited 2016 Sep 6];83(7). Available from: http://www.ncbi.nlm.nih.gov/pubmed/12098155

5. Nef T, Guidali M, Riener R. ARMin III - arm therapy exoskeleton with an ergonomic shoulder actuation. Appl Bionics Biomech. 2009;6(2):127-42.

6. Schiele A, Van Der Helm FCT. Kinematic design to improve ergonomics in human machine interaction. IEEE Trans Neural Syst Rehabil Eng. 2006;14(4):456-69.

7. Ludewig PM, Phadke V, Braman JP, Hassett DR, Cieminski CJ, Laprade RF. Motion of the shoulder complex during multiplanar humeral elevation. Vol. 91, Journal of Bone and Joint Surgery - Series A. 2009. p. 378-89.

8. Niyetkaliyev AS, Hussain S, Ghayesh MH, Alici G. Review on Design and Control Aspects of Robotic Shoulder Rehabilitation Orthoses. IEEE Transactions on Human-Machine Systems [Internet]. 2017 [cited 2017 Jun 13];1-12. Available from: http://ieeexplore.ieee.org/document/7932920/

9. Carignan C, Liszka M, Roderick S. Design of an arm exoskeleton with scapula motion for shoulder rehabilitation. In: 2005 International Conference on Advanced Robotics, ICAR '05, Proceedings [Internet]. Seattle, WA: IEEE; 2005. p. 524-31. Available from: https://ieeexplore.ieee.org/document/1507459

10. Mosadegh B, Polygerinos P, Keplinger C, Wennstedt S, Shepherd RF, Gupta U, et al. Pneumatic Networks for Soft Robotics that Actuate Rapidly. Adv Funct Mater [Internet]. 2014 Apr 10 [cited 2016 Jun 21];24(15):2163-70. Available from: http://doi.wiley.com/10.1002/adfm.201303288

11. Manti M, Hassan T, Passetti G, D’Elia N, Laschi C, Cianchetti M. A Bioinspired Soft Robotic Gripper for Adaptable and Effective Grasping. Soft Robot [Internet]. 2015 Sep [cited 2017 Jul 25];2(3):107-16. Available from: http://online.liebertpub.com/doi/10.1089/soro.2015.0009

630

12. Xiloyannis M, Cappello L, Binh KD, Antuvan CW, Masia L. Preliminary design and control of a soft exosuit for assisting elbow movements and hand grasping in activities of daily living. J Rehabil Assist Technol Eng. 2017;4:205566831668031. 
631

632

633

634

635

636

637

638

639

640

641

642

643

644

645

646

647

648

649

650

651

652

653

654

655

656

657

658

659

660

661

662

663

664

665

666

667

668
13. Seok S, Onal CD, Cho K-J, Wood RJ, Rus D, Kim S. Meshworm: A Peristaltic Soft Robot With Antagonistic Nickel Titanium Coil Actuators. IEEE/ASME Trans Mechatronics. 2013 Oct;18(5):1485-97.

14. Loepfe M, Schumacher CM, Lustenberger UB, Stark WJ. An Untethered, Jumping Roly-Poly Soft Robot Driven by Combustion. 2015;2(1):33-42.

15. Alouges F, DeSimone A, Giraldi L, Zoppello M. Can Magnetic Multilayers Propel Artificial Microswimmers Mimicking Sperm Cells? Soft Robot. 2015;2(3):117-28.

16. Shepherd RF, Ilievski F, Choi W, Morin SA, Stokes AA, Mazzeo AD, et al. Multigait soft robot. Proc Natl Acad Sci [Internet]. 2011;108(51):20400-3. Available from: http://www.pnas.org/cgi/doi/10.1073/pnas.1116564108

17. Galiana I, Hammond FL, Howe RD, Popovic MB. Wearable soft robotic device for poststroke shoulder rehabilitation: Identifying misalignments. In: 2012 IEEE/RSJ International Conference on Intelligent Robots and Systems [Internet]. IEEE; 2012. p. 317-22. Available from: http://ieeexplore.ieee.org/document/6385786/

18. Yap HK, Khin PM, Koh TH, Sun Y, Liang X, Lim JH, et al. A Fully Fabric-Based Bidirectional Soft Robotic Glove for Assistance and Rehabilitation of Hand Impaired Patients. IEEE Robot Autom Lett. 2017;2(3):1383-90.

19. Stilli A, Cremoni A, Bianchi M, Ridolfi A, Gerii F, Vannetti F, et al. AirExGlove-A novel pneumatic exoskeleton glove for adaptive hand rehabilitation in post-stroke patients. 2018 IEEE Int Conf Soft Robot RoboSoft 2018. 2018;579-84.

20. Fang J, Yuan J, Wang M, Xiao L, Yang J, Lin Z, et al. Novel Accordion-Inspired Foldable Pneumatic Actuators for Knee Assistive Devices. Soft Robot. 2019;00(00):1-14.

21. Ezzibdeh R, Arora P, Amanatullah DF. Utilization of a pneumatic exoskeleton after total knee arthroplasty. Arthroplast Today [Internet]. 2019;8-9. Available from: https://doi.org/10.1016/j.artd.2019.02.008

22. Sanchez RJ, Wolbrecht E, Smith R, Liu J, Rao S, Cramer S, et al. A pneumatic robot for retraining arm movement after stroke: Rationale and mechanical design. Vol. 2005, Proceedings of the 2005 IEEE 9th International Conference on Rehabilitation Robotics. 2005. p. 500-4.

23. Simpson CS, Okamura AM, Hawkes EW. Exomuscle: An inflatable device for shoulder abduction support. In: Proceedings - IEEE International Conference on Robotics and Automation [Internet]. IEEE; 2017 [cited 2017 Aug 25]. p. 6651-7. Available from: http://ieeexplore.ieee.org/document/7989785/

24. Natividad RF, Yeow CH. Development of a soft robotic shoulder assistive device for shoulder abduction. In: 2016 6th IEEE International Conference on Biomedical Robotics and Biomechatronics (BioRob) [Internet]. IEEE; 2016 [cited 2017 Sep 4]. p. 989-93. Available from: http://ieeexplore.ieee.org/document/7523758/

25. Oosterwijk AM, Nieuwenhuis MK, van der Schans CP, Mouton LJ. Shoulder and elbow range of motion for the performance of activities of daily living: A systematic review. Physiother 
26. Doorenbosch CAM, Harlaar J, Veeger D (H. EJ. The globe system: An unambiguous description of shoulder positions in daily life movements. J Rehabil Res Dev [Internet]. 2003;40(2):149. Available from: http://www.rehab.research.va.gov/jour/03/40/2/PDF/doorenbosch.pdf

27. Natividad RF, Del Rosario MRJ, Chen PCY, Yeow RCH. A Reconfigurable Pneumatic 17. Available from: http://www.liebertpub.com/doi/10.1089/soro.2017.0064

28. Natividad RF, Del Rosario MRJ, Chen PCY, Yeow RCH. A Hybrid Plastic-Fabric Soft 31. Reaz MBI, Hussain MS, Mohd-Yasin F. Techniques of EMG signal analysis: Detection,

32. Plagenhoef S, Gaynor Evans F, Abdelnour T, Evans FG, Abdelnour T. Anatomical Data for

30. Murgia A, Hortobágyi T, Wijnen A, Bruin L, Diercks R, Dekker R. Effects of age and sex on shoulder biomechanics and relative effort during functional tasks. J Biomech [Internet]. 2018;81:132-9. Available from: https://doi.org/10.1016/j.jbiomech.2018.10.001

Pneumatic Networks for Soft Robotics that Actuate Rapidly. Adv Funct Mater [Internet]. 2014 Apr 10 [cited 2016 Jun 21];24(15):2163-70. Available from: http://doi.wiley.com/10.1002/adfm.201303288

Bending Actuator with Reconfigurable Bending Profiles. In: Proceedings - IEEE International Conference on Robotics and Automation [Internet]. Singapore: IEEE; 2017 [cited 2017 Aug 21]. p. 6700-5. Available from: http://ieeexplore.ieee.org/document/7989792/

29. Mosadegh B, Polygerinos P, Keplinger C, Wennstedt S, Shepherd RF, Gupta U, et al. processing, classification and applications. Biol Proced Online. 2006;8(1):11-35.

Analyzing Human Motion. Res Q Exerc Sport [Internet]. 1983;54(2):169-78. Available from: http://www.tandfonline.com/doi/abs/10.1080/02701367.1983.10605290

33. Rehmat N, Zuo J, Meng W, Liu Q, Xie SQ, Liang H. Upper limb rehabilitation using robotic exoskeleton systems: a systematic review. Int J Intell Robot Appl [Internet]. 2018;(0123456789). Available from: http://link.springer.com/10.1007/s41315-018-0064-8

34. Kadivar Z, Beck CE, Rovekamp RN, Malley MKO, Joyce CA. On the Efficacy of Isolating Shoulder and Elbow Movements with a Soft, Portable, and Wearable Robotic Device.

35. Neill CTO, Phipps NS, Cappello L, Paganoni S, Walsh CJ, O’Neill CTCT, et al. A soft wearable robot for the shoulder: Design, characterization, and preliminary testing. IEEE Int Conf Rehabil Robot [Internet]. 2017 Jul [cited 2017 Aug 15];02129:1672-8. Available from: http://ieeexplore.ieee.org/document/8009488/

36. Tiseni L, Xiloyannis M, Chiaradia D, Lotti N, Solazzi M, Kooij H Van Der, et al. On the edge between soft and rigid : an assistive shoulder exoskeleton with hyper-redundant kinematics. 2019 IEEE 16th Int Conf Rehabil Robot. 2019;618-24.

37. Xiloyannis M, Chiaradia D, Frisoli A, Masia L. Physiological and kinematic effects of a soft 

exosuit on arm movements. J Neuroeng Rehabil. 2019;16(1):1-15.

708

\section{$71416 \quad$ Figure Legends and Tables}

\section{Figure 1:}

716 Front (Left) and back (Right) views of the 2-DOF shoulder exoskeleton. The exoskeleton is powered

717 by 2 pairs of antagonistic, modular actuators. The elevation/depression pair and the steering pair are 718 attached perpendicularly.

\section{Figure 2:}

720 A) A completed spine with a completed module. The yellow and white, 3-D printed structures are

721 clearly seen on the spine and module respectively. The red band on the module indicates the location

722 of the heat-seal seam. The actuator's geometric properties are shown: [d] Module Spacing, [L]

723 Module Length, [W] Module Width. (B) The assembled actuator. The spine is attached to neoprene

724 through industrial grade hook-and-loop fasteners. Fabric straps are placed across to limit unwanted

725 inflation at the center. (C) A CAD representation of the 3-D printed structures (D) The actuator curls

726 during inflation. The strap and neoprene are removed for clarity. At this pose, all the modules are in

727 point contact with adjacent modules. (E) The actuator curls into a helix through the application of

728 external forces. Its ability to perform 3-D bending is clearly demonstrated. 
730 (A) The actuator as represented by the physical model. The model considers the inflated modules as

731 extruded ellipses Model segments are color coded. Red: angled section. Blue: straight middle sections.

732 Yellow: Edge sections. (B) The top view. The definition of the bending angle, $\theta$, is shown; a straight

733 actuator corresponds to an angle of $0^{\circ}$. (C): The free body diagram showing how forces are applied to

734 the module and to the arm. (D) A visual representation of the mathematical expressions used to

735 determine the contact area. The modules revolve around a point $\mathrm{Oj}$ [green]. The line projection of the

736 rectangular contact area is shown [orange].

\section{Figure 4:}

738 The range of motion of the actuator is shown for an exoskeleton with attached D2 variants. (A)

739 Abduction or adduction. The yellow area highlights non-bending modules that lengthen the moment

740 arm of the bending modules. (B) Rotation along the plane of elevation. (C) Forward flexion or

741 extension. Alternatively, a combination of these movements can be performed through the combined

742 activation of actuators. (D-F) The exoskeleton performing reaching actions. The positions shown

743 were achieved in a single motion, starting from the neutral position.

\section{Figure 5:}

745 (A) The two-axis torque measurement system. RED: The A-A' axis. Orange: The B-B' axis. (B) A

746 top view of a schematic representation of the actuator. A straight actuator corresponds to an A-A'

747 angle of $0^{\circ}$ while a fully folded actuator corresponds to $-180^{\circ}$. (C) A front view of the actuator. The

748 definition of the B-B' angle is shown.

$749 \quad$ Figure 6: 
sEMG and IMU sensors were placed on the subjects. (A) Front View. (B) Side View (C) Back View.

751 (D) Subjects were asked to perform abduction and adduction, horizontal flexion and extension, and

752 forward flexion and extension under various conditions. The (yellow) casings housing the IMUs are

753 shown. LD-Lateral Deltoid, AD-Anterior Deltoid, P-Pectoralis Major, I-Infraspinatus, PD-Posterior

754 Deltoid, Abd-Abduction, Add-Adduction, FF-Forward Flexion, FE-Forward Extension, HF-

755 Horizontal Flexion, HE-Horizontal Extension

\section{Figure 7:}

757 The experiment results for the actuator (A) Torque output as a function of A-A' angle when pressurized 758 to $80 \mathrm{kPa}$. (B) Torque output at various inflation pressures, at an A-A' angle set to $90^{\circ}$. Solid lines

759 represent the output of the model. (C) A contour plot of torque output at various A-A'angles and B-B'

760 angles for the D2 variant. (D) The waveform in response to a 60s square wave, with 0-80kPa peak-to-

761 peak amplitudes. (E) The averaged step response during inflation. (F) The averaged step response 762 during deflation.

\section{Figure 8:}

764 Segmented trajectory and muscle activation data sets extracted from a single subject. Representative 765 muscles were chosen for visual illustration. (A) Abduction (Lateral Deltoid) and Adduction (Lateral

766 Deltoid). (B) Horizontal Flexion (Anterior Deltoid) and Horizontal Extension (Posterior Deltoid). (C)

767 Forward Flexion (Anterior Deltoid) and Forward Extension (Posterior Deltoid). Muscle activation

768 waveforms show a direct correlation with the corresponding trajectory. There is an apparent offset 769 between free, unpowered and powered phases.

\section{Figure 9:}


771 Box plots summarizing muscle activation data for slow movements. Lat Delt-Lateral Deltoid. Ant

772 Delt-Anterior Deltoids. Pos Delt-Posterior Deltoid. Pecs-Pectoralis Major. Infra-Infraspinatus

773 Figure 10:

774 Box plots summarizing muscle activation data for fast movements. Lat Delt-Lateral Deltoid. Ant

775 Delt-Anterior Deltoids. Pos Delt-Posterior Deltoid. Pecs-Pectoralis Major. Infra-Infraspinatus

$77617 \quad$ Additional Materials

$777 \quad 17.1$ Additional File 1

778 - Format: .docx

779 - Title: Supplementary Information

780 - Description: Non-essential data and text that further enriches the reader understand the work

$781 \quad$ presented.

$782 \quad 17.2$ Additional File 2

783 - Format: .mp4

784 - Title: Range of Motion Demo

785 - Description: Video presenting capabilities of the exoskeleton 


\section{Figures}

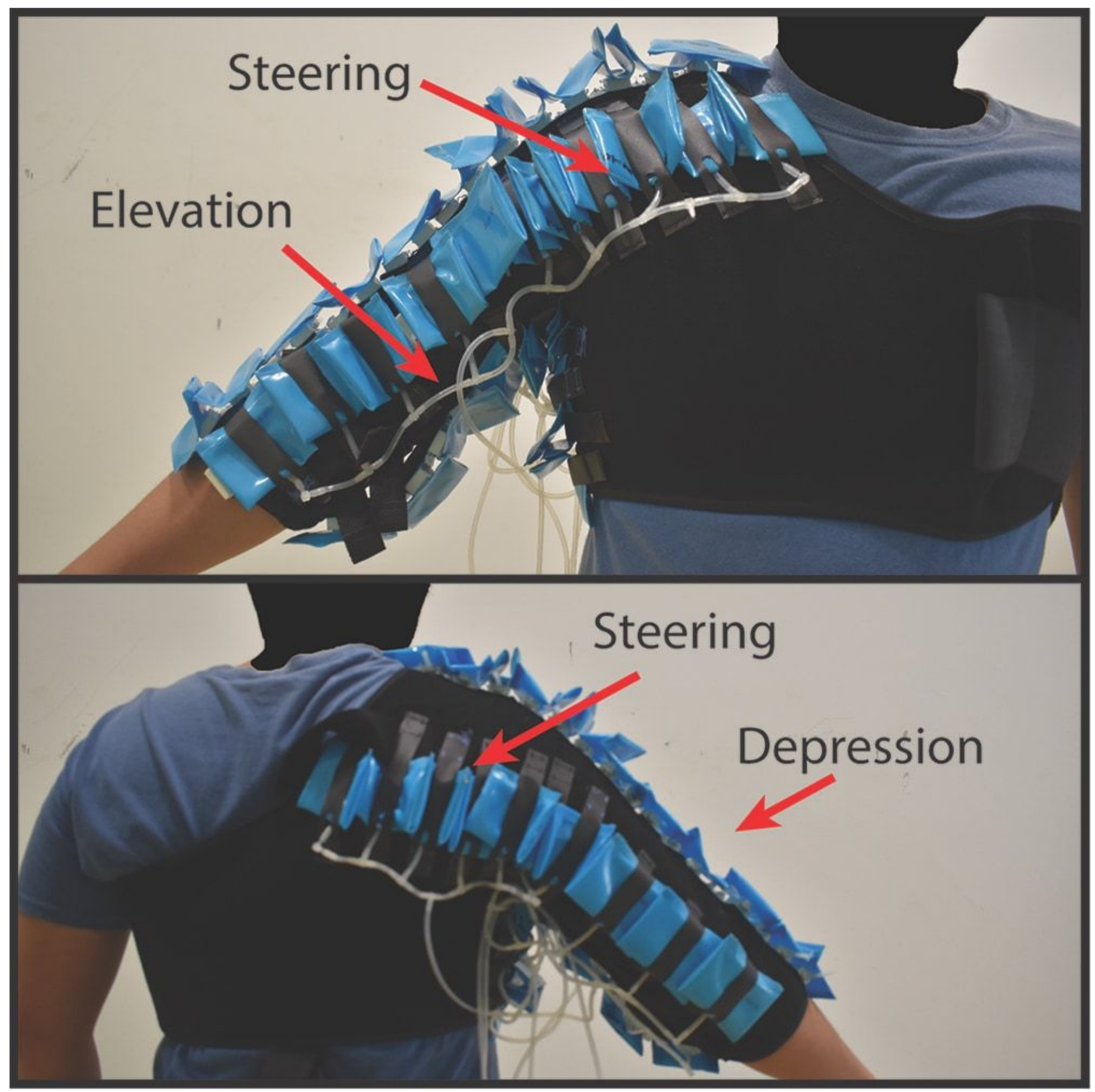

\section{Figure 1}

Front (Left) and back (Right) views of the 2-DOF shoulder exoskeleton. The exoskeleton is powered by 2 pairs of antagonistic, modular actuators. The elevation/depression pair and the steering pair are attached perpendicularly. 


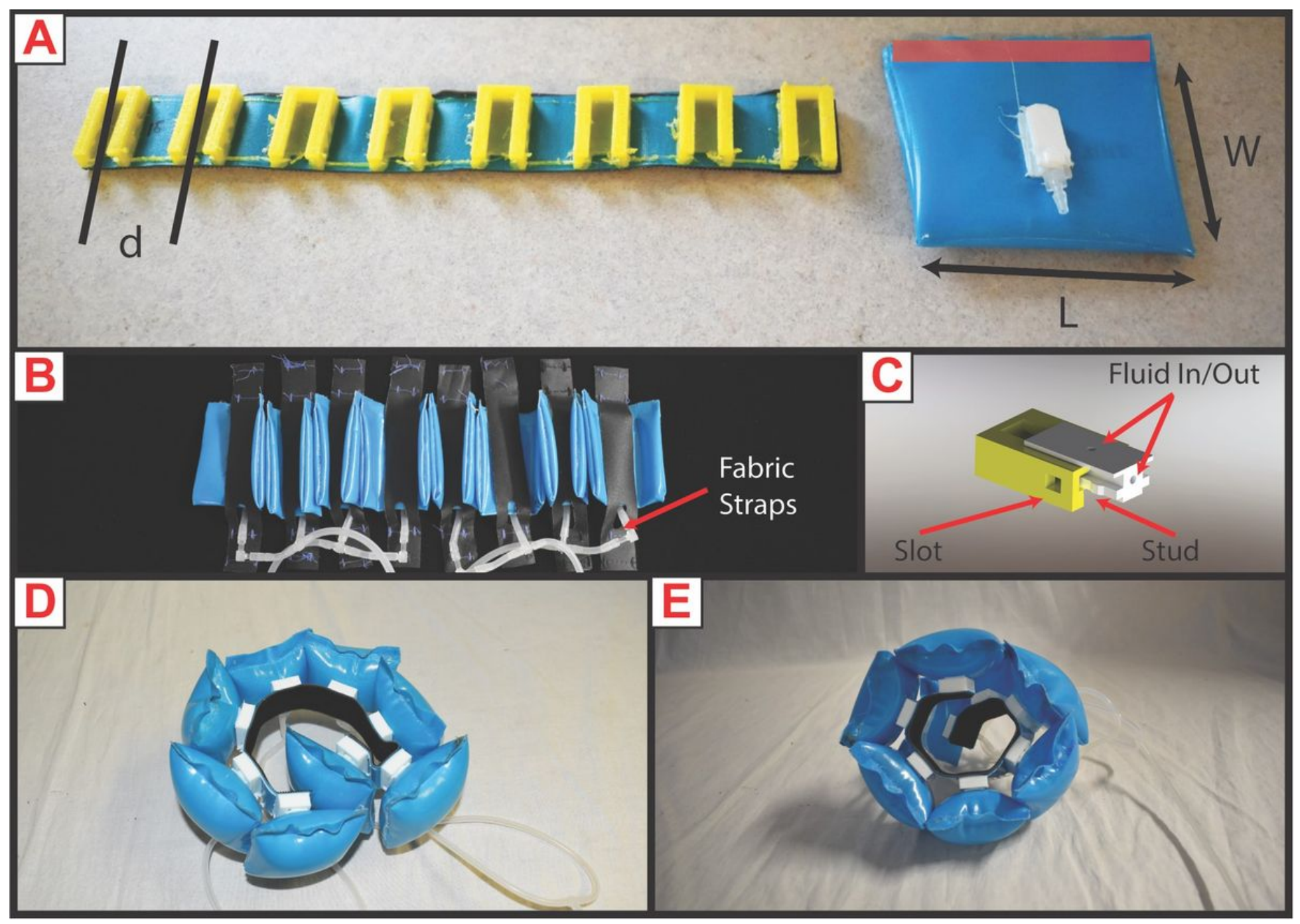

\section{Figure 2}

A) A completed spine with a completed module. The yellow and white, 3-D printed structures are clearly seen on the spine and module respectively. The red band on the module indicates the location of the heatseal seam. The actuator's geometric properties are shown: [d] Module Spacing, [L] Module Length, [W] Module Width. (B) The assembled actuator. The spine is attached to neoprene through industrial grade hook-and-loop fasteners. Fabric straps are placed across to limit unwanted inflation at the center. (C) A CAD representation of the 3-D printed structures (D) The actuator curls during inflation. The strap and neoprene are removed for clarity. At this pose, all the modules are in point contact with adjacent modules. (E) The actuator curls into a helix through the application of external forces. Its ability to perform 3-D bending is clearly demonstrated. 

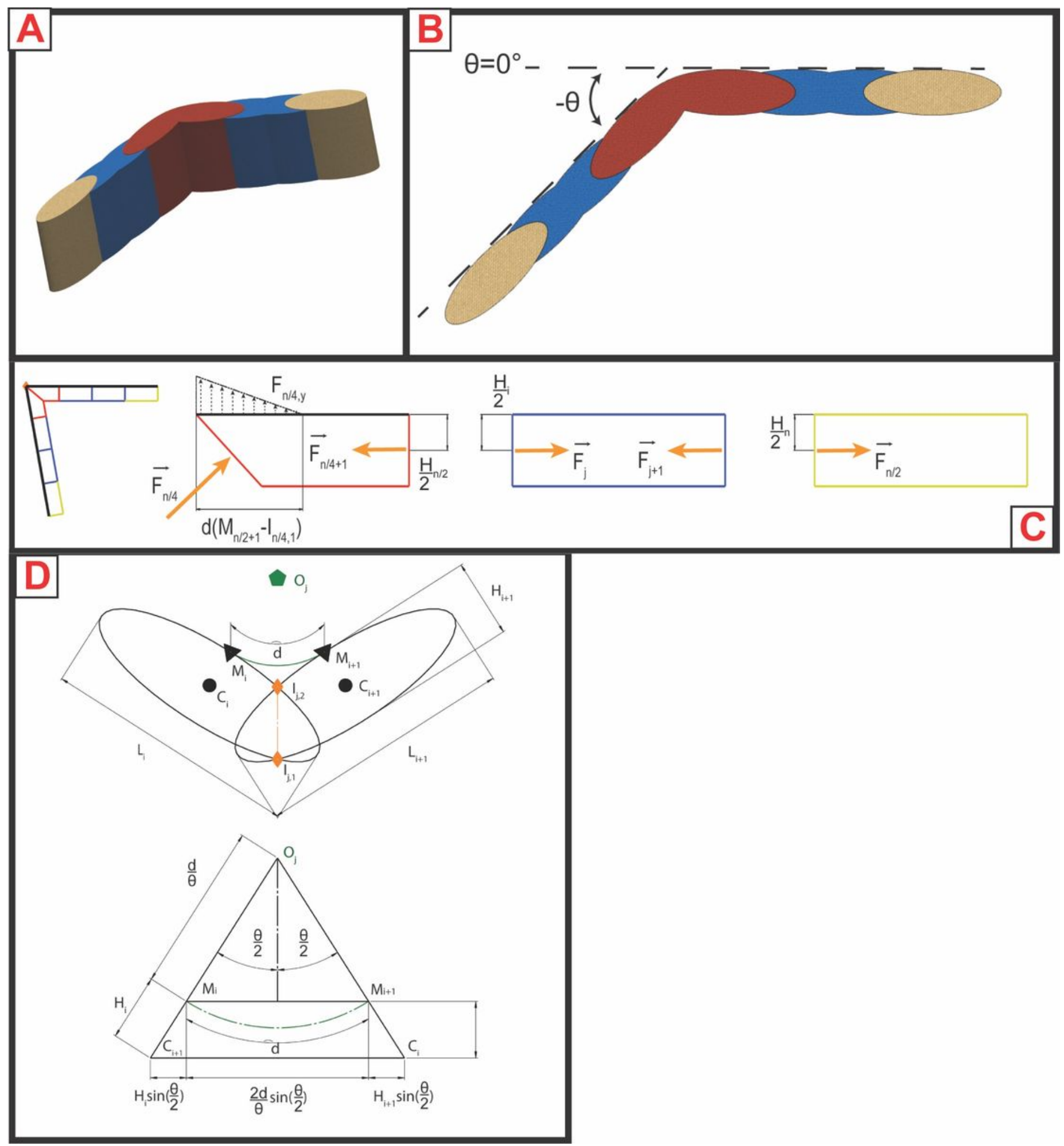

Figure 3

(A) The actuator as represented by the physical model. The model considers the inflated modules as extruded ellipses Model segments are color coded. Red: angled section. Blue: straight middle sections. Yellow: Edge sections. (B) The top view. The definition of the bending angle, $\theta$, is shown; a straight actuator corresponds to an angle of $0^{\circ}$. (C): The free body diagram showing how forces are applied to the module and to the arm. (D) A visual representation of the mathematical expressions used to determine 
the contact area. The modules revolve around a point Oj [green]. The line projection of the rectangular contact area is shown [orange].

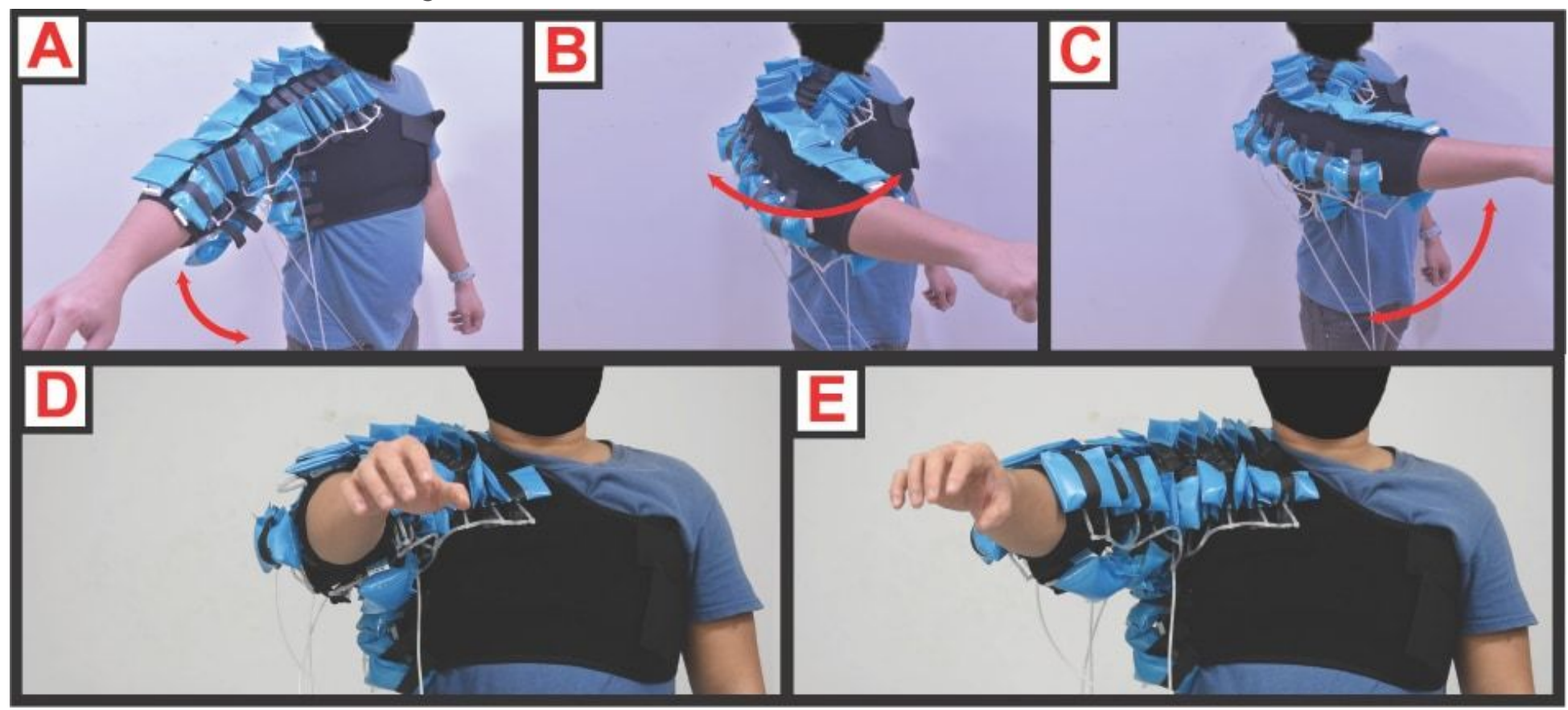

Figure 4

The range of motion of the actuator is shown for an exoskeleton with attached D2 variants. (A) Abduction or adduction. The yellow area highlights non-bending modules that lengthen the moment arm of the bending modules. (B) Rotation along the plane of elevation. (C) Forward flexion or extension. Alternatively, a combination of these movements can be performed through the combined activation of actuators. (D-F) The exoskeleton performing reaching actions. The positions shown were achieved in a single motion, starting from the neutral position.

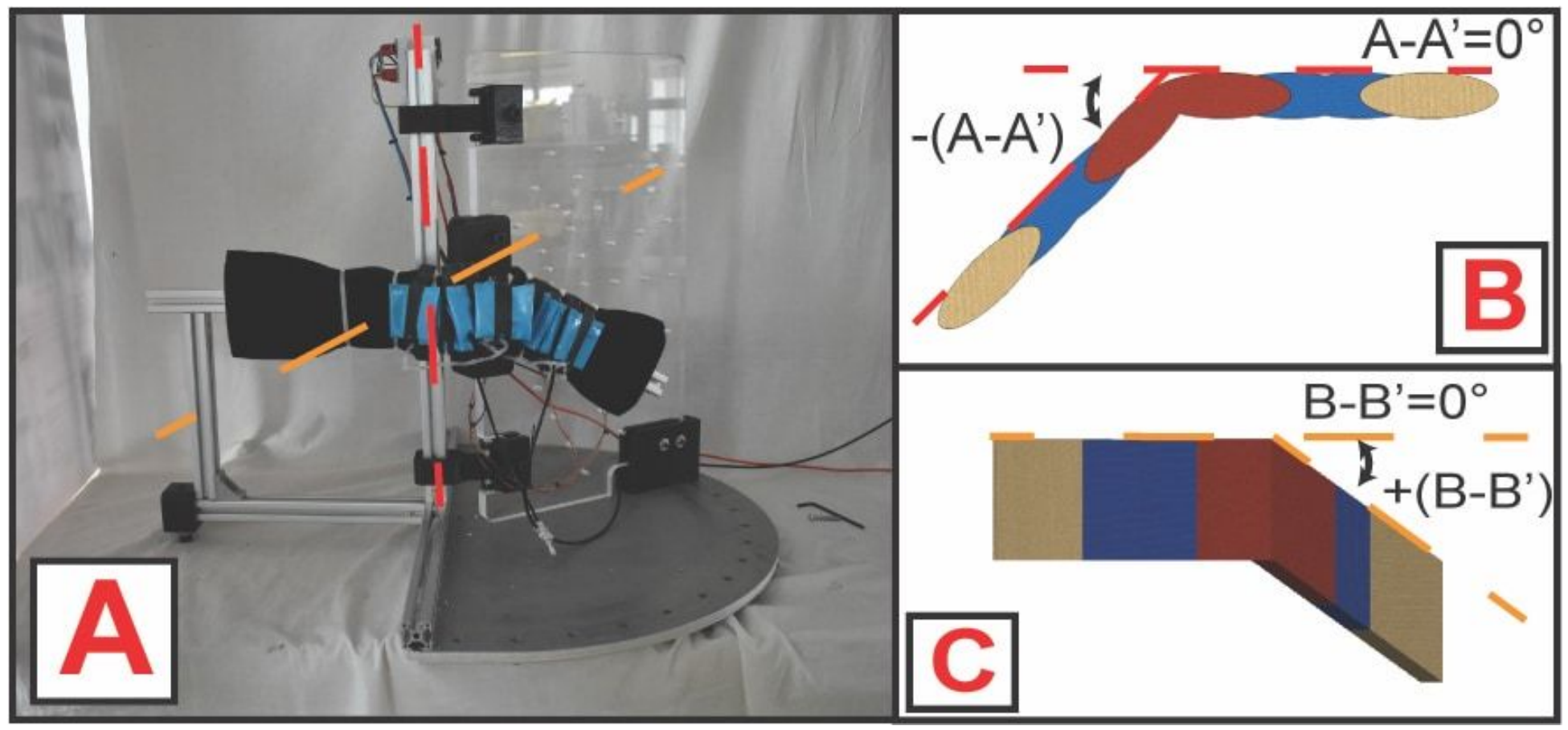




\section{Figure 5}

(A) The two-axis torque measurement system. RED: The A-A' axis. Orange: The B-B' axis. (B) A top view of a schematic representation of the actuator. A straight actuator corresponds to an A-A' angle of $0^{\circ}$ while a fully folded actuator corresponds to $-180^{\circ}$. (C) A front view of the actuator. The definition of the B-B' angle is shown.

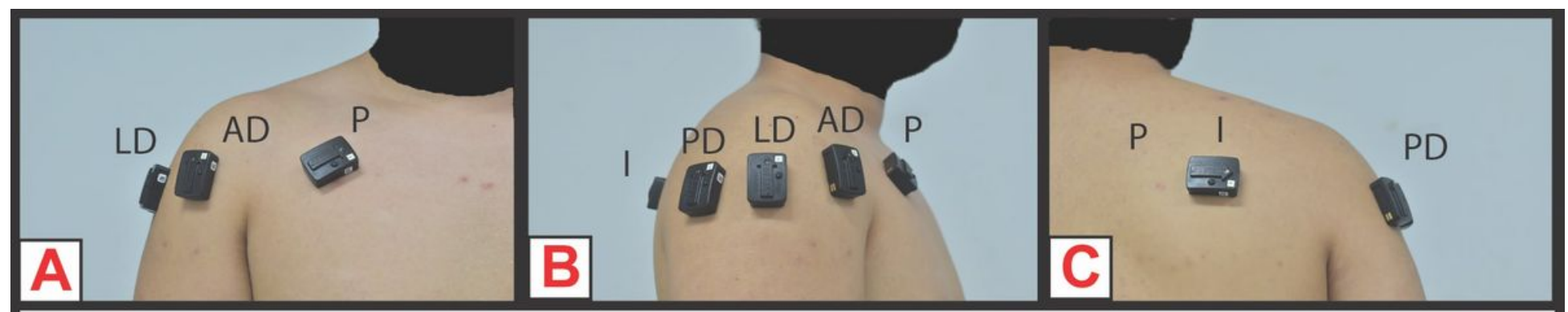

D
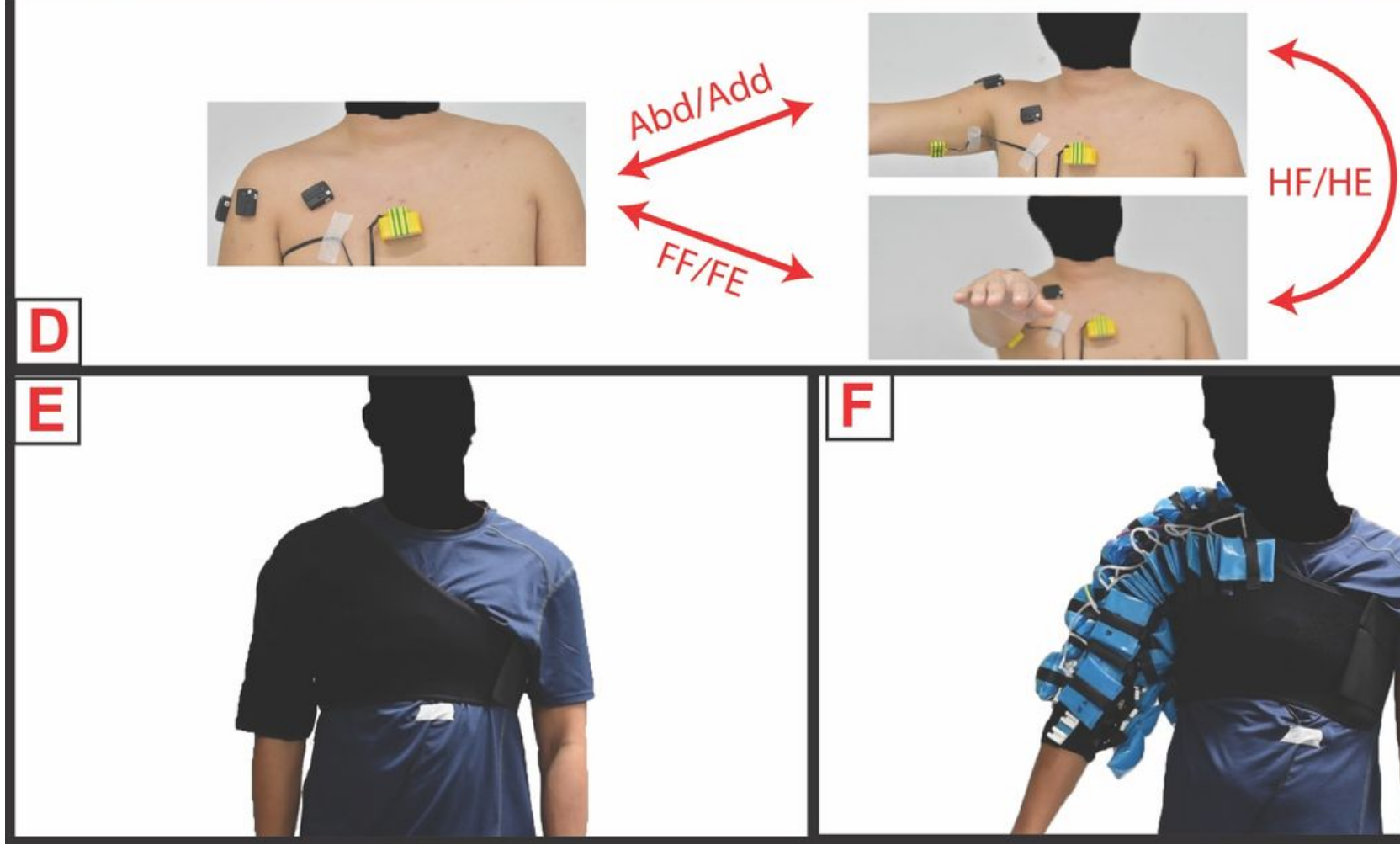

\section{$\mathrm{F}$}

\section{Figure 6}

sEMG and IMU sensors were placed on the subjects. (A) Front View. (B) Side View (C) Back View. (D) Subjects were asked to perform abduction and adduction, horizontal flexion and extension, and forward flexion and extension under various conditions. The (yellow) casings housing the IMUs are shown. LDLateral Deltoid, AD-Anterior Deltoid, P-Pectoralis Major, I-Infraspinatus, PD-Posterior Deltoid, AbdAbduction, Add-Adduction, FF-Forward Flexion, FE-Forward Extension, HF-Horizontal Flexion, HEHorizontal Extension 


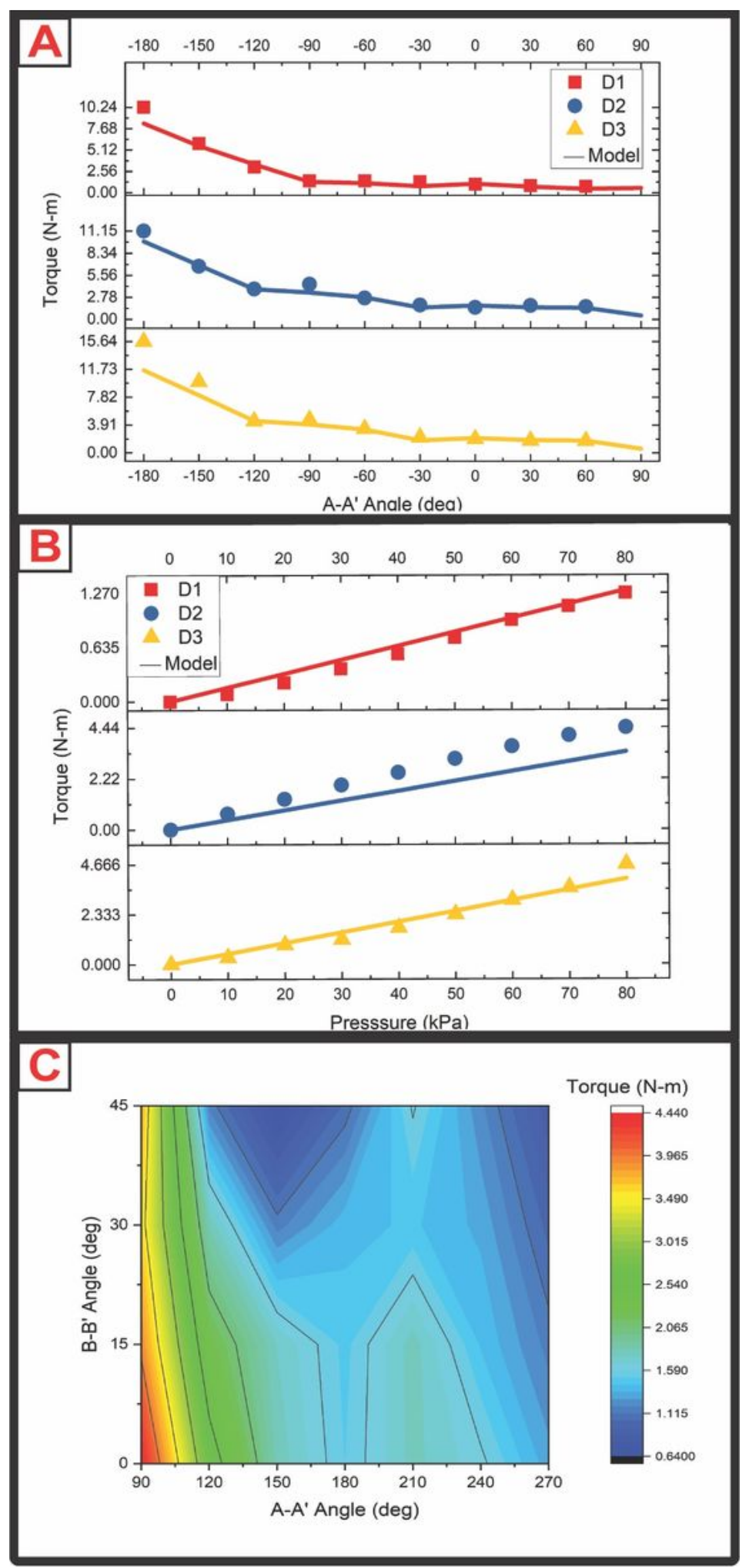

\section{Figure 7}

The experiment results for the actuator (A) Torque output as a function of $A-A^{\prime}$ angle when pressurized to $80 \mathrm{kPa}$. (B) Torque output at various inflation pressures, at an A-A' angle set to $90^{\circ}$. Solid lines represent the output of the model. (C) A contour plot of torque output at various A-A'angles and B-B' angles for the D2 variant. (D) The waveform in response to a 60 s square wave, with 0-80kPa peak-to-peak amplitudes. (E) The averaged step response during inflation. (F) The averaged step response during deflation. 


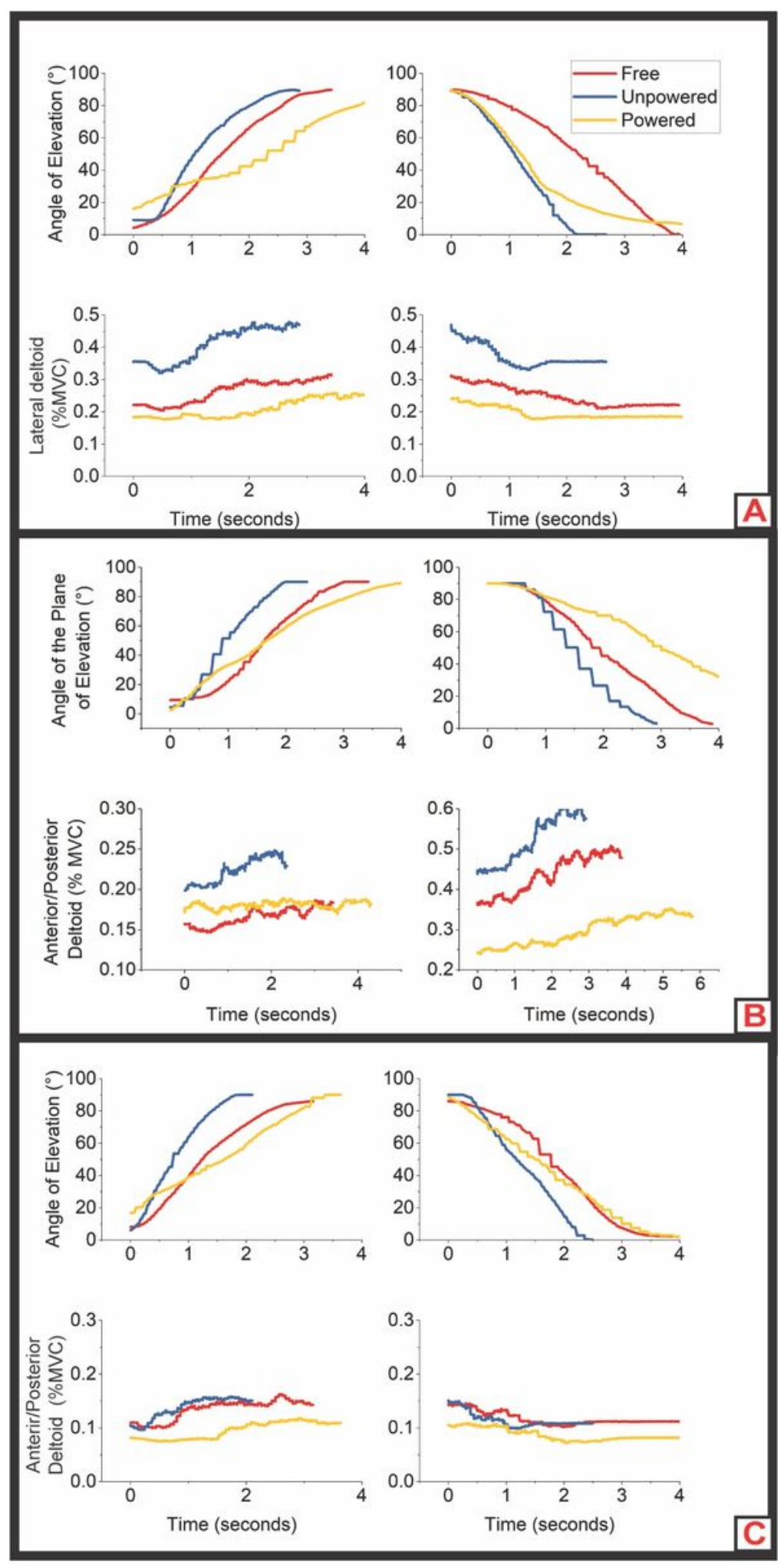

\section{Figure 8}

Segmented trajectory and muscle activation data sets extracted from a single subject. Representative muscles were chosen for visual illustration. (A) Abduction (Lateral Deltoid) and Adduction (Lateral Deltoid). (B) Horizontal Flexion (Anterior Deltoid) and Horizontal Extension (Posterior Deltoid). (C) Forward Flexion (Anterior Deltoid) and Forward Extension (Posterior Deltoid). Muscle activation 
waveforms show a direct correlation with the corresponding trajectory. There is an apparent offset between free, unpowered and powered phases.

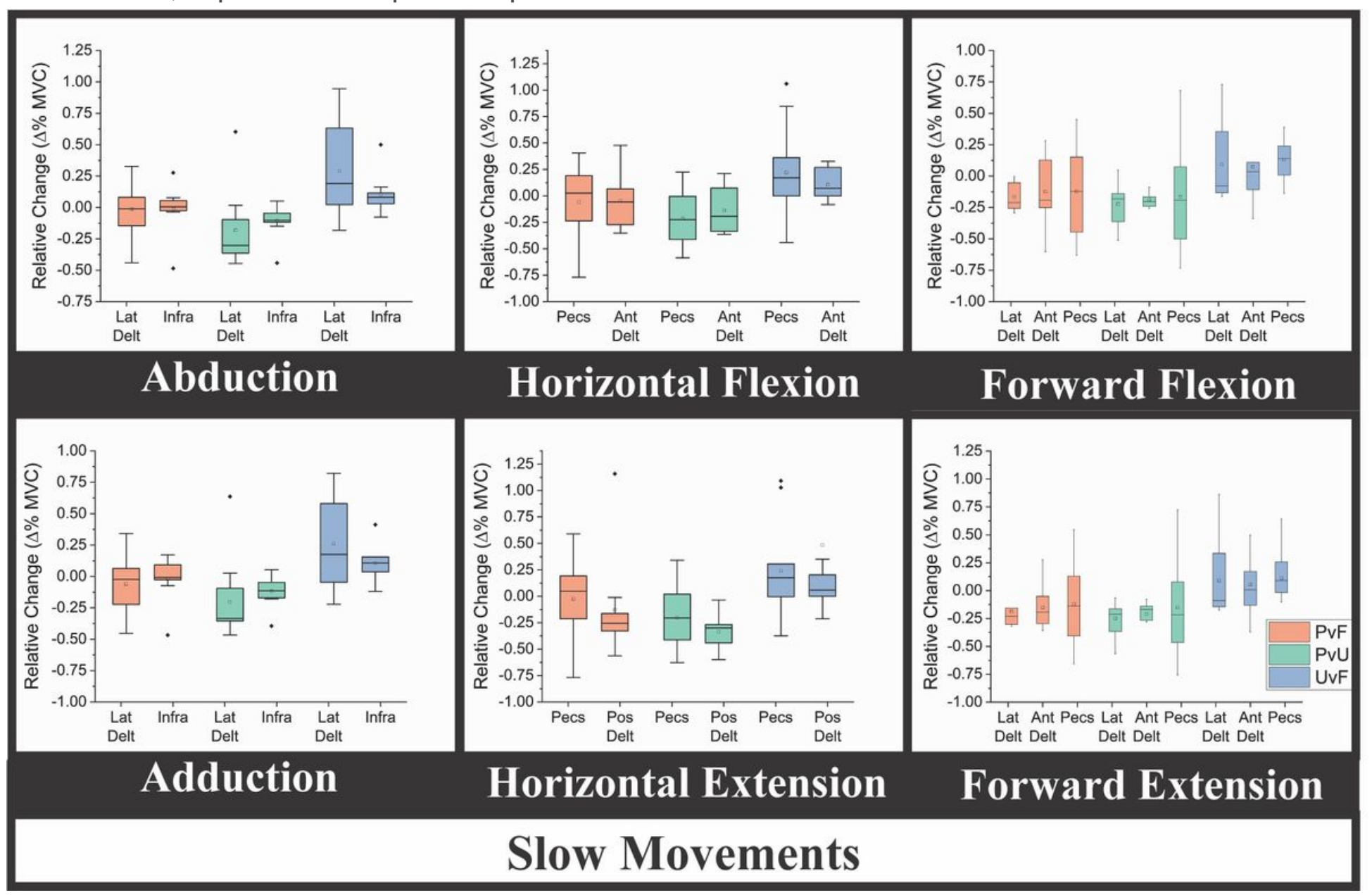

Figure 9

Box plots summarizing muscle activation data for slow movements. Lat Delt-Lateral Deltoid. Ant DeltAnterior Deltoids. Pos Delt-Posterior Deltoid. Pecs-Pectoralis Major. Infra-Infraspinatus 


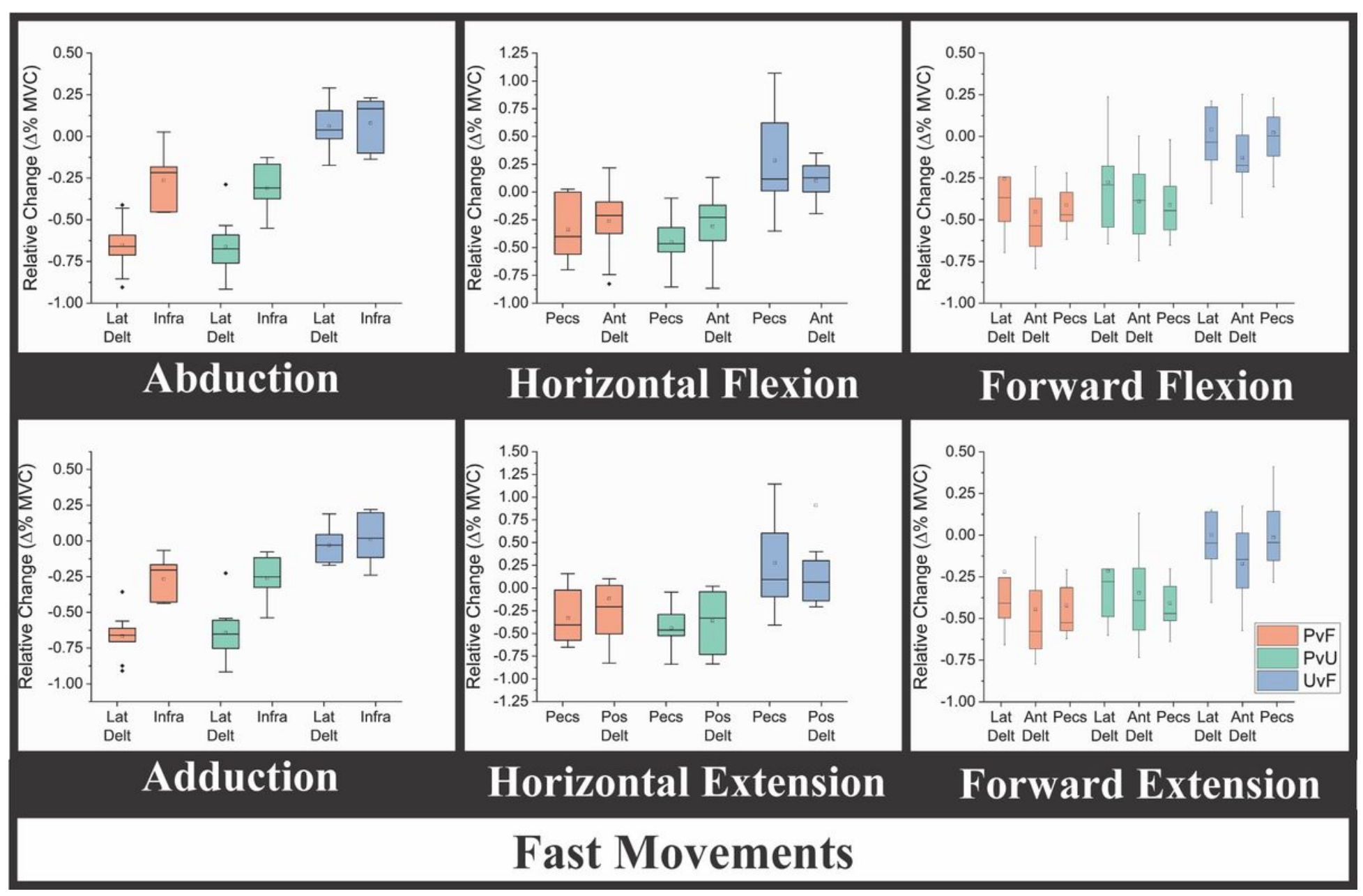

Figure 10

Box plots summarizing muscle activation data for fast movements. Lat Delt-Lateral Deltoid. Ant DeltAnterior Deltoids. Pos Delt-Posterior Deltoid. Pecs-Pectoralis Major. Infra-Infraspinatus

\section{Supplementary Files}

This is a list of supplementary files associated with this preprint. Click to download.

- AdditionalFile2.mp4

- AdditionalFile1R1.docx 